\title{
Supplementary
}

\section{Formamidinium Haloplumbate Intermediates: the Missing Link in a Chain of Hybrid Perovskites Crystallization}

\author{
Andrey A. Petrov ${ }^{1,4^{\dagger}}$, Sergey A. Fateev ${ }^{1+}$, Victor N. Khrustalev ${ }^{2,3}$, Li Yumao ${ }^{4}$, Pavel V. Dorovatovskii ${ }^{5}$, \\ Yan V. Zubavichus ${ }^{6}$, Eugene A. Goodilin ${ }^{1,7}$, Alexey B. Tarasov ${ }^{1,4^{*}}$ \\ ${ }^{1}$ Laboratory of New Materials for Solar Energetics, Faculty of Materials Science, Lomonosov Moscow State \\ University, 1 Lenin Hills, 119991 Moscow, Russian Federation \\ ${ }^{2}$ Inorganic Chemistry Department, Peoples' Friendship University of Russia (RUDN University), 6 Miklukho- \\ Maklay, 117198, Moscow, Russia \\ ${ }^{3}$ N.D. Zelinsky Institute of Organic Chemistry of Russian Academy of Sciences, 47 Leninsky Prosp., 119991 \\ Moscow, Russia \\ ${ }^{4}$ Faculty of Materials Science, Shenzhen MSU-BIT University, Longgang District, Ruyi Road, 299, 518572 \\ Shenzhen, China \\ ${ }^{5}$ National Research Centre 'Kurchatov Institute', 1 Acad. Kurchatov Sq., 123182, Moscow, Russia \\ ${ }^{6}$ Federal Research Center Boreskov Institute of Catalysis, Lavrentiev Ave. 5, 630090, Novosibirsk, Russia \\ ${ }^{7}$ Department of Chemistry, Lomonosov Moscow State University, 1 Lenin Hills, 119991 Moscow, Russian \\ Federation \\ ${ }^{\dagger}$ These authors contributed equally
}




\section{Crystal Structure of the Obtained Intermediate Phases}

$(\mathrm{FA})_{2} \mathrm{~Pb}_{3} \mathrm{I}_{8} \cdot 4 \mathrm{DMF}$
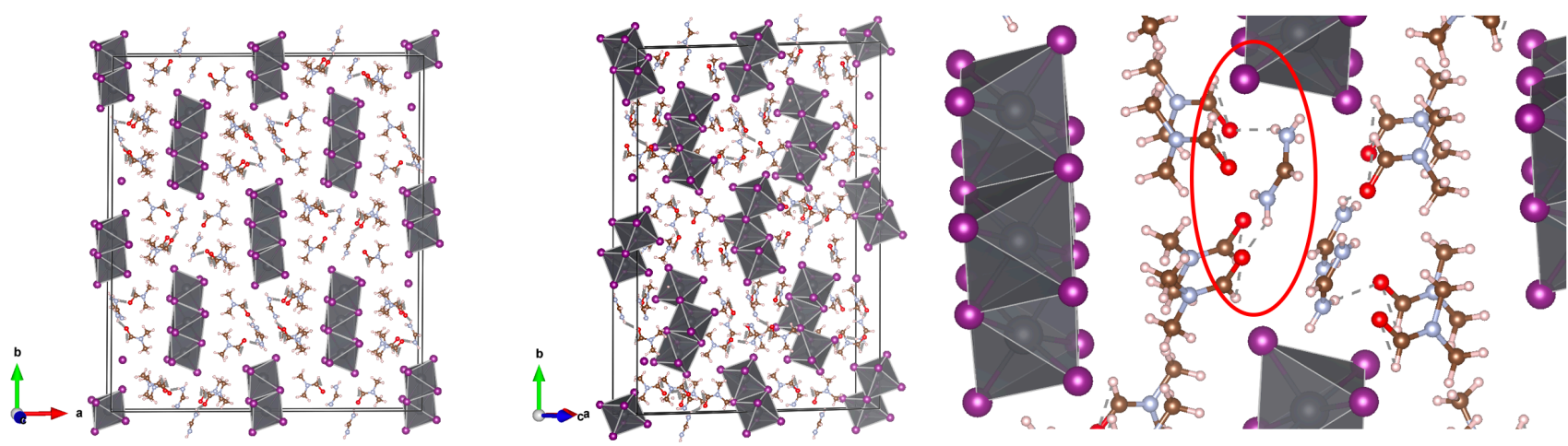

The inorganic lattice of the $\left.(\mathrm{FA})_{2} \mathrm{~Pb}_{3}\right|_{8}$.4DMF structure contains $\left[\left.\mathrm{Pb}_{3}\right|_{8}\right]$ ribbons of edge-connected octahedra oriented along $\mathrm{C}$ axis of the unit cell. These ribbons are isostructural to similar $\left[\left.\mathrm{Pb}_{3}\right|_{8}\right]$ ribbons in the structures of $\left.(\mathrm{MA})_{2} \mathrm{~Pb}_{3}\right|_{8} \cdot 2 \mathrm{DMF}$ and $(\mathrm{MA})_{2} \mathrm{~Pb}_{3} \mathrm{l}_{8} \cdot 2 \mathrm{DMSO}$, however arranged in a different manner so that they leave larger voids for cations and solvent molecules than the last two structures. In the voids between the ribbons, the formamidinium cations and DMF molecules are arranged together so that each $\mathrm{FA}^{+}$cation is connected by hydrogen bonds with oxygen atoms of two adjacent DMF molecules. We assume that it is the $\mathrm{FA}^{+}$cation ability to coordinate two DMF molecules on both sides without steric hindrance which explains the fact that $\mathrm{Pb}$-excessive adduct with formamidinium contains 2 times more DMF molecules in the structure then MA-containing counterpart.

$(\mathrm{MA})_{2} \mathrm{~Pb}_{3} \mathrm{I}_{8} \cdot 2 \mathrm{DMF}$ (for comparison):
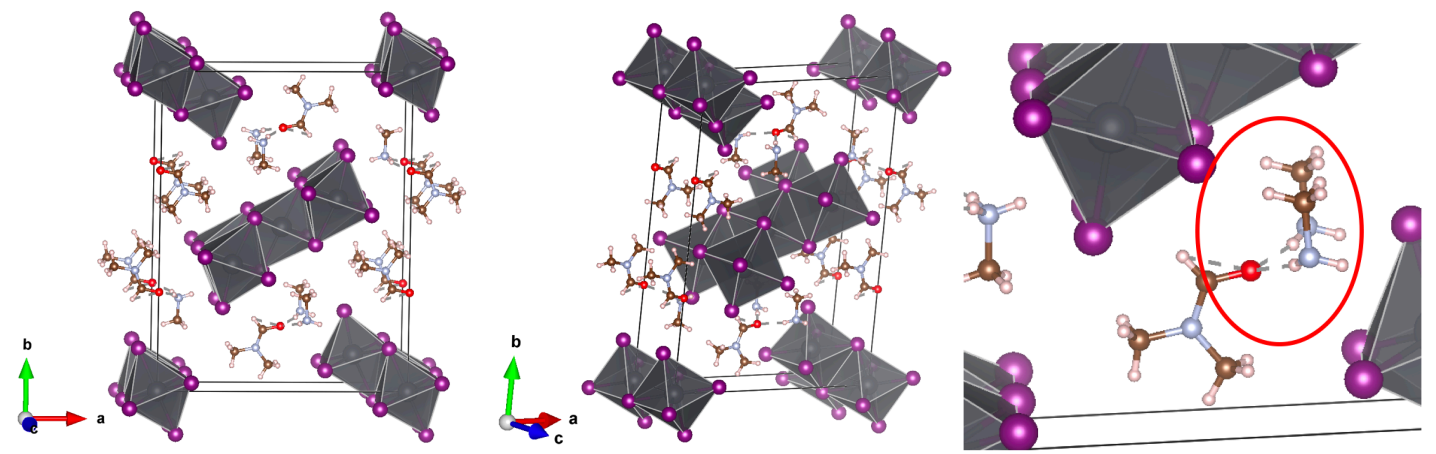

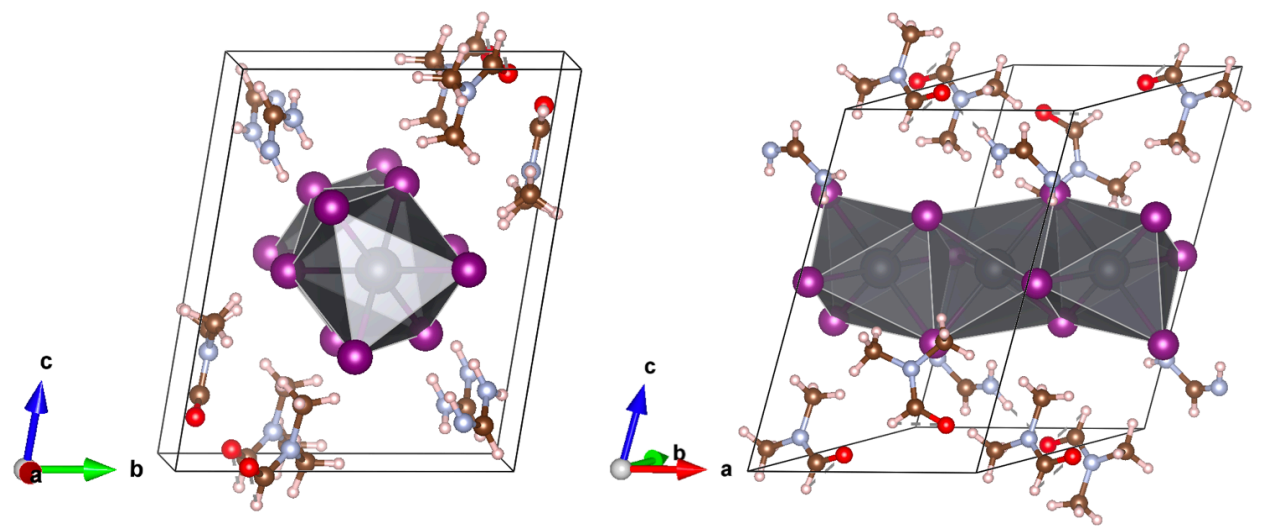

The structure of $\mathrm{FAPb}_{3} \cdot 2 \mathrm{DMF}$ is formed by chains of face-shared $\mathrm{Pbl}_{6}$ octahedra along the 100 direction. Each chain is surrounded by $\mathrm{FA}^{+}$cation and DMF molecules connected to each other similarly to the case of $(\mathrm{FA})_{2} \mathrm{~Pb}_{3} \mathrm{I}_{8}$.4DMF. The structure is also additionally stabilized by $(\mathrm{N}) \mathrm{H} \cdots \mathrm{I}$ hydrogen bonds between $\mathrm{FA}^{+}$ cation and iodine atoms of the chains.

$(\mathrm{FA})_{5} \mathrm{~Pb}_{2} \mathrm{I}_{9} \cdot 0.5 \mathrm{DMSO}$

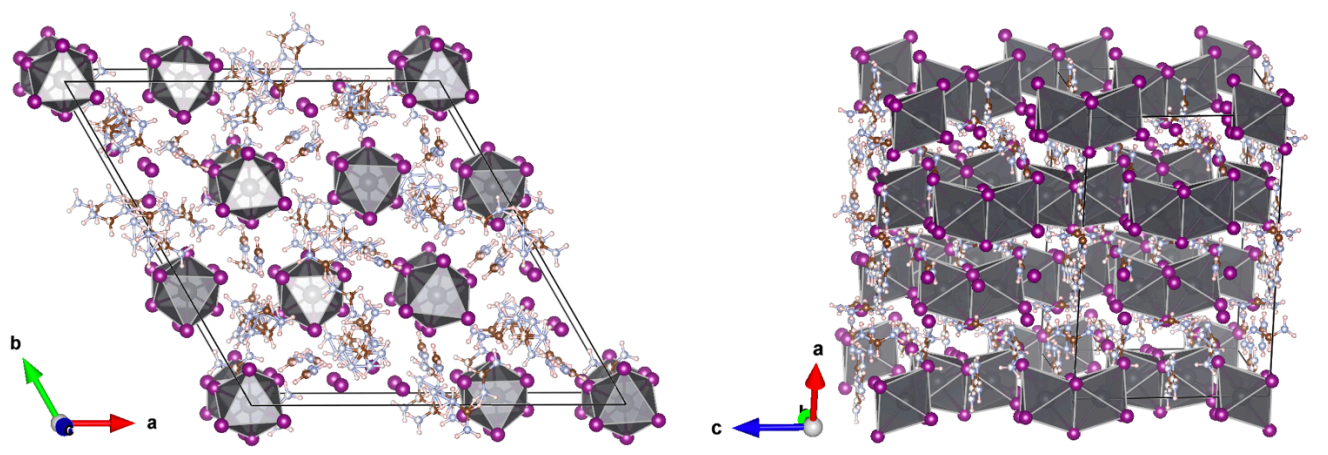

The structure of $(\mathrm{FA})_{5} \mathrm{~Pb}_{2} \mathrm{l}_{9} \cdot 0.5 \mathrm{DMSO}$ contains isolated $\mathrm{Pb}_{2} \mathrm{l}_{9}$ dimers separated by partially disordered $\mathrm{FA}^{+}$ cations. The $\mathrm{Pb}_{2} \mathrm{l}_{9}$ dimers have hexagonal arrangement with respect to each other. We assume, that the overall structure is stabilized by relatively short $(\mathrm{N}) \mathrm{H} \cdots \mathrm{I}$ hydrogen bonds between dimers and organic cations. 

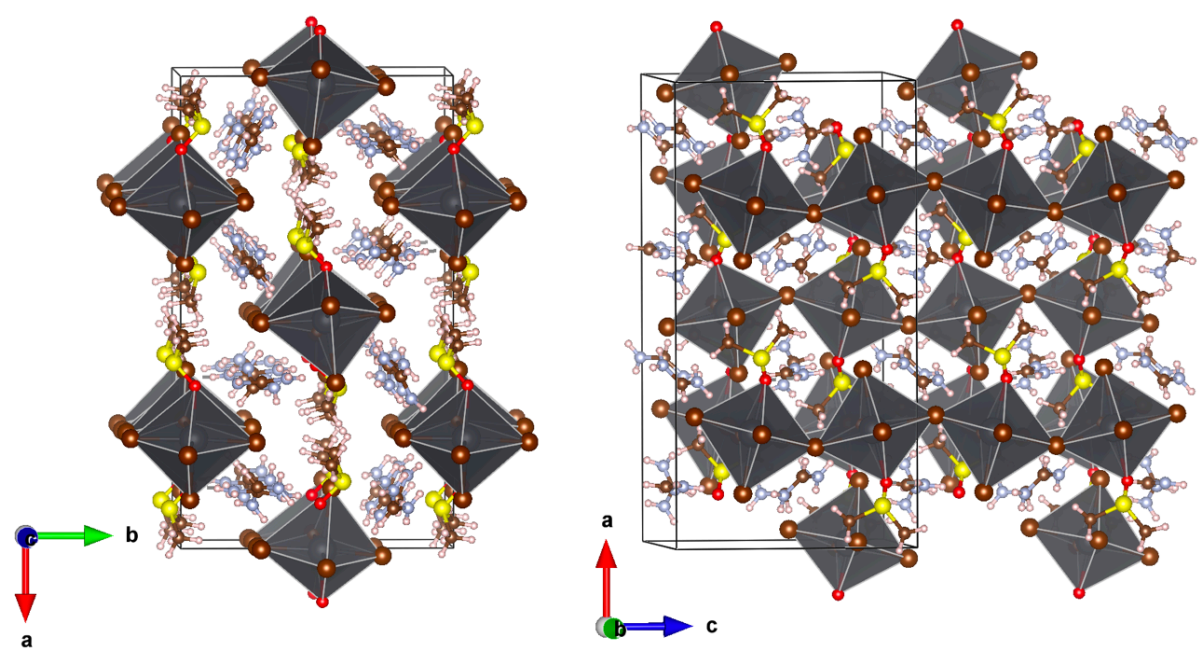

The structure of $\mathrm{FA}_{2} \mathrm{PbBr}_{4} \cdot \mathrm{DMSO}$ adduct can be represented as chains of corner-shared $\left[\mathrm{PbBr}_{6}\right]$ octahedra in which one of the non-bridging bromine atoms is replaced by a DMSO molecule. Thus, the $\mathrm{Pb}^{2+}$ ions remain six-fold coordination, however, one of the atoms of their first coordination sphere is the oxygen atom of DMSO. There are two different (S)O $\cdots \mathrm{Pb}$ bonds in the structure: $2.52 \AA$ and $2.61 \AA$ which are slightly longer than that in the known structure of $\mathrm{PbBr}_{2} \cdot 2 \mathrm{DMSO}$ adduct. The chains of $\left[(\mathrm{DMSO}) \mathrm{PbBr}_{5}\right]$ are held together by hydrogen bonds with formamidinium cations, where each cation simultaneously forms bonds with two adjacent chains. 
Table S1. General crystallographic information on samples studied.

\begin{tabular}{|c|c|c|c|c|}
\hline Crystal code & $(\mathrm{FA})_{2} \mathrm{~Pb}_{3} \mathrm{I}_{8} \cdot 4 \mathrm{DMF}$ & $\mathrm{FAPbl}_{3} \cdot 2 \mathrm{DMF}$ & $(\mathrm{FA})_{5} \mathrm{~Pb}_{2} \mathrm{I}_{9} \cdot 0.5 \mathrm{DMSO} *$ & (FA) ${ }_{2} \mathrm{PbBr}_{4} \cdot \mathrm{DMSO}$ \\
\hline Empirical formula & $\mathrm{C}_{14} \mathrm{H}_{38} \mathrm{I}_{8} \mathrm{~N}_{8} \mathrm{O}_{4} \mathrm{~Pb}_{3}$ & $\mathrm{C}_{7} \mathrm{H}_{19} \mathrm{l}_{3} \mathrm{~N}_{4} \mathrm{O}_{2} \mathrm{~Pb}$ & $\mathrm{C}_{6} \mathrm{H}_{28}{ }_{9} \mathrm{~N}_{10} \mathrm{O}_{0.5} \mathrm{~Pb}_{2} \mathrm{~S}_{0.5}$ & $\mathrm{C}_{4} \mathrm{H}_{16} \mathrm{Br}_{4} \mathrm{~N}_{4} \mathrm{OPbS}$ \\
\hline$f w$ & 2019.32 & 779.16 & 1820.90 & 695.90 \\
\hline$T, \mathrm{~K}$ & $100.0(2)$ & $100.0(2)$ & $100.0(2)$ & $100.0(2)$ \\
\hline Crystal size, $\mathrm{mm}$ & $0.02 \times 0.02 \times 0.15$ & $0.03 \times 0.03 \times 0.15$ & $0.03 \times 0.06 \times 0.10$ & $0.02 \times 0.07 \times 0.12$ \\
\hline Crystal system & Orthorhombic & Triclinic & Hexagonal & Orthorhombic \\
\hline Space group & Fdd2 & $P-1$ & $P-6$ & $\mathrm{PCa}_{1}$ \\
\hline$a, \AA$ & $40.200(8)$ & $7.8788(16)$ & $26.770(3)$ & $22.334(5)$ \\
\hline$b, \AA$ & $45.720(9)$ & $10.497(2)$ & $26.770(3)$ & $12.933(3)$ \\
\hline$c, \AA$ & $4.5800(9)$ & $12.678(3)$ & $14.860(2)$ & $11.629(2)$ \\
\hline$\alpha,^{\circ}$ & 90 & $75.92(3)$ & 90 & 90 \\
\hline$\beta, \circ$ & 90 & $75.11(3)$ & 90 & 90 \\
\hline$\gamma_{1}^{\circ}$ & 90 & $68.82(3)$ & 120 & 90 \\
\hline$V, \AA^{3}$ & $8418(3)$ & $931.6(4)$ & 9222.44 & $3359.0(12)$ \\
\hline$Z$ & 8 & 8 & 9 & 8 \\
\hline $\mathrm{d}_{\mathrm{c},} \mathrm{g} \mathrm{cm}^{-3}$ & 3.187 & 2.778 & 2.951 & 2.749 \\
\hline$F\left(\begin{array}{lll}0 & 0 & 0\end{array}\right)$ & 7040 & 692 & 7083 & 2512 \\
\hline$\mu, \mathrm{mm}^{-1}$ & 22.142 & 17.453 & 19.136 & 24.819 \\
\hline$\theta_{\text {max }}{ }^{\circ}$ & 30.852 & 30.865 & 30.867 & 30.878 \\
\hline Index range & $\begin{array}{l}-50<h<50 \\
-58<k<58 \\
-5<1<5\end{array}$ & $\begin{array}{l}-10<h<10 \\
-13<k<13 \\
-16<l<16\end{array}$ & $\begin{array}{l}-34<h<34 \\
-34<k<34 \\
-19<1<19\end{array}$ & $\begin{array}{l}-28<h<28 \\
-16<k<16 \\
-14<1<14\end{array}$ \\
\hline $\begin{array}{l}\text { No. of reflections } \\
\text { collected }\end{array}$ & 16271 & 15000 & 79929 & 32520 \\
\hline $\begin{array}{l}\text { Data/restraints/ } \\
\text { parameters }\end{array}$ & $4532 / 1 / 168$ & $4035 / 0 / 162$ & $13829 / 54 / 235$ & $7275 / 1 / 277$ \\
\hline$R_{1} ; \mathrm{w} R_{2}(I>2 \sigma(I))$ & $0.0445 ; 0.1014$ & $0.0537 ; 0.1263$ & $0.1230 ; 0.2654$ & $0.0566 ; 0.1339$ \\
\hline$R_{1} ; \mathrm{w} R_{2}$ (all data) & $0.0480 ; 0.1036$ & $0.0592 ; 0.1303$ & $0.1510 ; 0.2866$ & $0.0616 ; 0.1399$ \\
\hline $\begin{array}{l}\text { Goodness-of-fit } \\
\text { (GOF) on } F^{2}\end{array}$ & 0.849 & 0.951 & 1.049 & 0.821 \\
\hline$T_{\min } ; T_{\max }$ & $0.120 ; 0.600$ & $0.140 ; 0.560$ & $0.212 ; 0.555$ & $0.120 ; 0.600$ \\
\hline$\Delta \rho_{\max } ; \Delta \rho_{\text {min }}, \mathrm{e}^{-3}$ & $3.081 ;-2.235$ & $2.934 ;-3.490$ & $5.532 ;-3.757$ & $2.477 ;-2.837$ \\
\hline Data completeness & $99.1 \%$ & $98.8 \%$ & $99.3 \%$ & $99.4 \%$ \\
\hline
\end{tabular}

* According to PLATON's SQUEEZE procedure, the volume of solvent accessible voids in the structure is $560.3 \AA^{3}$ and 202.6ē per unit cell, which roughly corresponds to 0.5 DMSO molecules per formula unit. 
Table S2. Phases observed upon crystallization of solutions with various compositions* .

\begin{tabular}{|c|c|c|}
\hline & DMF & DMSO \\
\hline $\mathrm{MAPbBr}_{3}$ & $\mathrm{MAPbBr}_{3}$ & $\mathrm{MAPbBr}_{3}$ \\
\hline $\operatorname{MAPb}\left(\mathrm{I}_{0.25} \mathrm{Br}_{0.75}\right)_{3}$ & $\begin{array}{c}(\mathrm{MA})_{2} \mathrm{~Pb}_{3}\left(\mathrm{I}_{1-\mathrm{x}} \mathrm{Br} \mathrm{rr}_{\mathrm{x}}\right)_{8} * 2 \mathrm{DMF} \\
\mathrm{MAPb}\left(\mathrm{I}_{1-\mathrm{x}} \mathrm{Br} \mathrm{r}_{\mathrm{x}}\right)_{3}\end{array}$ & $\begin{array}{c}(\mathrm{MA})_{2} \mathrm{~Pb}_{3}\left(\mathrm{I}_{1-\mathrm{x}} \mathrm{Br} \mathrm{r}_{\mathrm{x}}\right)_{8} * 2 \mathrm{DMSO} \\
\mathrm{MAPb}\left(\mathrm{I}_{1-\mathrm{x}} \mathrm{Br} \mathrm{r}_{\mathrm{x}}\right)_{3}\end{array}$ \\
\hline $\operatorname{MAPb}\left(\mathrm{I}_{0.5} \mathrm{Br}_{0.5}\right)_{3}$ & $\begin{array}{c}(\mathrm{MA})_{2} \mathrm{~Pb}_{3}\left(\mathrm{I}_{1-\mathrm{x}} \mathrm{Br} \mathrm{Br}_{\mathrm{x}}\right)_{8} * 2 \mathrm{DMF} \\
\mathrm{MAPb}\left(\mathrm{I}_{1-\mathrm{x}} \mathrm{Br} \mathrm{r}_{\mathrm{x}}\right)_{3}\end{array}$ & $\begin{array}{c}(\mathrm{MA})_{2} \mathrm{~Pb}_{3}\left(\mathrm{I}_{1-\mathrm{x}} \mathrm{Br} \mathrm{r}_{\mathrm{x}}\right)_{8} * 2 \mathrm{DMSO} \\
\operatorname{MAPb}\left(\mathrm{I}_{1-\mathrm{x}} \mathrm{Br}_{\mathrm{x}}\right)_{3}\end{array}$ \\
\hline $\operatorname{MAPb}\left(\mathrm{I}_{0.75} \mathrm{Br}_{0.25}\right)_{3}$ & $\begin{array}{c}(\mathrm{MA})_{2} \mathrm{~Pb}_{3}\left(\mathrm{I}_{1-\mathrm{x}} \mathrm{Br} \mathrm{Br}_{\mathrm{x}}\right)_{8} * 2 \mathrm{DMF} \\
\operatorname{MAPb}\left(\mathrm{I}_{1-\mathrm{x}} \mathrm{Br} \mathrm{r}_{\mathrm{x}}\right)_{3}\end{array}$ & $\begin{array}{c}(\mathrm{MA})_{2} \mathrm{~Pb}_{3}\left(\mathrm{I}_{1-\mathrm{x}} \mathrm{Br} \mathrm{r}_{\mathrm{x}}\right)_{8} * 2 \mathrm{DMSO} \\
\mathrm{MAPb}\left(\mathrm{I}_{1-\mathrm{x}} \mathrm{Br}_{\mathrm{x}}\right)_{3}\end{array}$ \\
\hline $\mathrm{MAPbl}_{3}$ & $\begin{array}{l}(\mathrm{MA})_{2} \mathrm{~Pb}_{3} \mathrm{I}_{8} * 2 \mathrm{DMF} \\
(\mathrm{MA})_{2} \mathrm{~Pb}_{2} \mathrm{I}_{6} * 2 \mathrm{DMF}\end{array}$ & $\begin{array}{c}(\mathrm{MA})_{2} \mathrm{~Pb}_{3} \mathrm{l}_{8} * 2 \mathrm{DMSO} \\
\mathrm{Pbl}_{2} * 2 \mathrm{DMSO}\end{array}$ \\
\hline $\mathrm{FA}_{0.25} \mathrm{MA}_{0.75} \mathrm{Pbl}_{3}$ & $\begin{array}{c}(\mathrm{MA})_{2} \mathrm{~Pb}_{3} \mathrm{l}_{8} * 2 \mathrm{DMF} \\
\alpha-\left(\mathrm{MA}_{x} \mathrm{FA}_{1-\mathrm{x}}\right) \mathrm{Pbl}_{3}\end{array}$ & $\begin{array}{c}(\mathrm{MA})_{2} \mathrm{~Pb}_{3} \mathrm{I}_{8} * 2 \mathrm{DMSO} \\
\mathrm{Pbl}_{2} * 2 \mathrm{DMSO}\end{array}$ \\
\hline $\mathrm{FA}_{0.5} \mathrm{MA}_{0.5} \mathrm{Pbl}_{3}$ & $\begin{array}{c}(\mathrm{MA})_{2} \mathrm{~Pb}_{3} \mathrm{I}_{8} * 2 \mathrm{DMF} \\
\alpha-\left(\mathrm{MA}_{\mathrm{x}} \mathrm{FA}_{1-\mathrm{x}}\right) \mathrm{Pbl}_{3} \\
\delta-\mathrm{FAPb}_{3}\end{array}$ & $\begin{array}{c}(\mathrm{MA})_{2} \mathrm{~Pb}_{3} \mathrm{I}_{8} * 2 \mathrm{DMSO} \\
\mathrm{Pbl}_{2} * 2 \mathrm{DMSO}\end{array}$ \\
\hline $\mathrm{FA}_{0.75} \mathrm{MA}_{0.25} \mathrm{Pbl}_{3}$ & $\begin{array}{c}(\mathrm{MA})_{2} \mathrm{~Pb}_{3} \mathrm{I}_{8} * 2 \mathrm{DMF} \\
\alpha-\left(\mathrm{MA}_{\mathrm{x}} \mathrm{FA}_{1-\mathrm{x}}\right) \mathrm{Pbl}_{3} \\
\delta-\mathrm{FAPbl}_{3}\end{array}$ & $\begin{array}{c}(\mathrm{MA})_{2} \mathrm{~Pb}_{3} \mathrm{I}_{8} * 2 \mathrm{DMSO} \\
\mathrm{Pbl}_{2} * 2 \mathrm{DMSO}\end{array}$ \\
\hline $\mathrm{FAPbl}_{3}$ & $\begin{array}{l}(\mathrm{FA})_{2} \mathrm{~Pb}_{3} 1_{8} * 4 \mathrm{DMF} \\
(\mathrm{FA})_{2} \mathrm{~Pb}_{2} 1_{6} * 4 \mathrm{DMF}\end{array}$ & $\begin{array}{l}\mathrm{Pbl}_{2} * 2 \mathrm{DMSO} \\
\delta-\mathrm{FAPbl}_{3}\end{array}$ \\
\hline $\operatorname{FAPb}\left(\mathrm{I}_{0.75} \mathrm{Br}_{0.25}\right)_{3}$ & $\begin{array}{c}(\mathrm{MA})_{2} \mathrm{~Pb}_{3} \mathrm{I}_{8} * 2 \mathrm{DMF} \\
(\mathrm{MA})_{2} \mathrm{~Pb}_{2} \mathrm{I}_{6} * 2 \mathrm{DMF} \\
\delta-\mathrm{FAPbl}_{3}\end{array}$ & amorphous product \\
\hline $\mathrm{FAPb}\left(\mathrm{I}_{0.5} \mathrm{Br}_{0.5}\right)_{3}$ & $\begin{array}{c}(\mathrm{MA})_{2} \mathrm{~Pb}_{3} \mathrm{l}_{8} * 2 \mathrm{DMF}(?) \\
(\mathrm{MA})_{2} \mathrm{~Pb}_{2} \mathrm{I}_{6} * 2 \mathrm{DMF}(?) \\
\delta-\left.\mathrm{FAPb}\right|_{3}\end{array}$ & amorphous product \\
\hline $\operatorname{FAPb}\left(\mathrm{I}_{0.25} \mathrm{Br}_{0.75}\right)_{3}$ & $\operatorname{FAPb}\left(I_{1-x} \mathrm{Br}_{x}\right)_{3}$ & $\begin{array}{c}\mathrm{d}-\mathrm{FAPbl} \mathrm{I}_{3} \\
\mathrm{FAPb}\left(\mathrm{I}_{1-\mathrm{x}} \mathrm{Br}_{\mathrm{x}}\right)_{3}\end{array}$ \\
\hline $\mathrm{FAPbBr}_{3}$ & $\mathrm{FAPbBr}_{3}$ & $\mathrm{FAPbBr}_{3}$ \\
\hline
\end{tabular}

*26 series of in-situ XRD measurements are given below. 


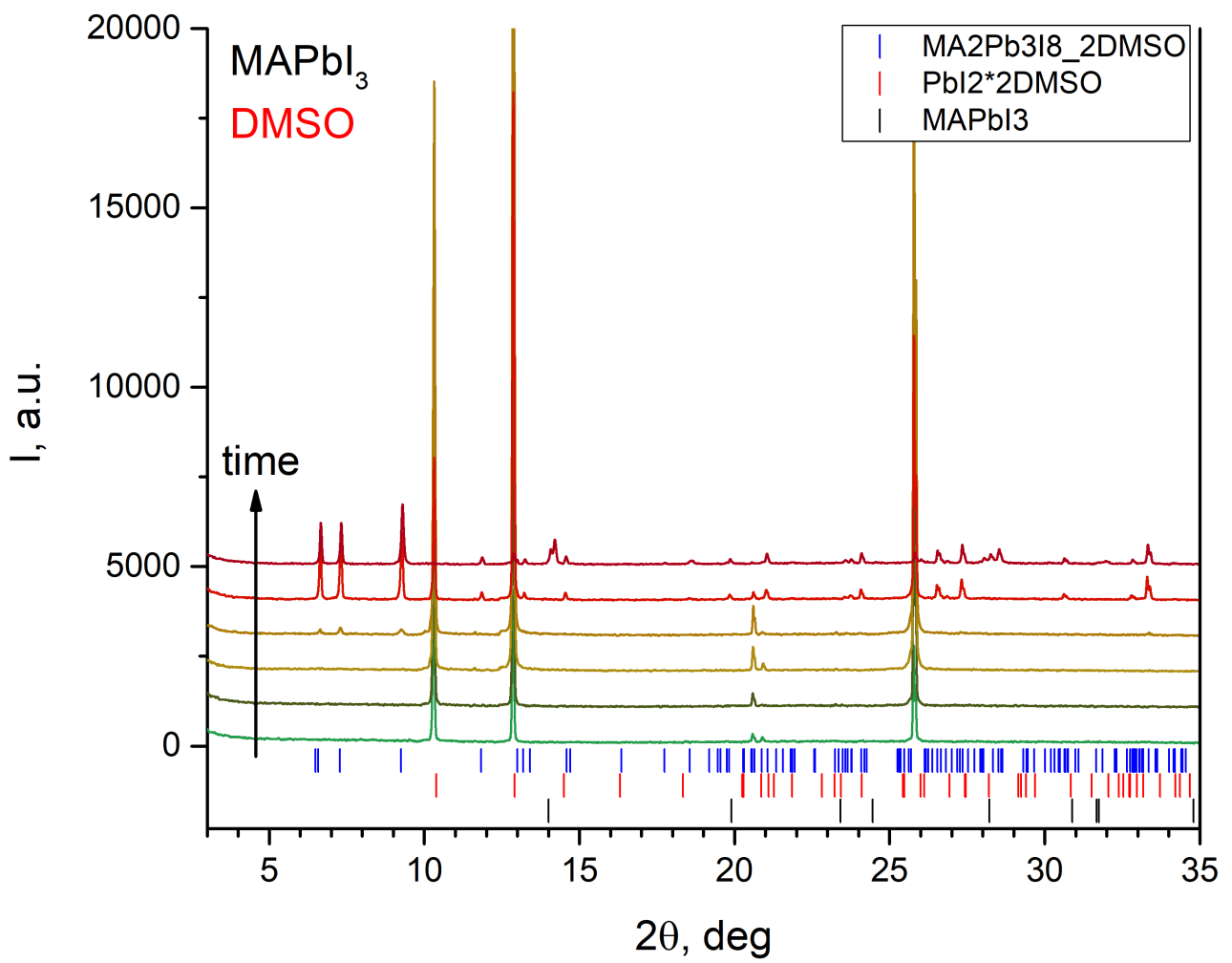

Figure S1. Crystallization of $\mathrm{MAPbl}_{3}$ from DMSO.

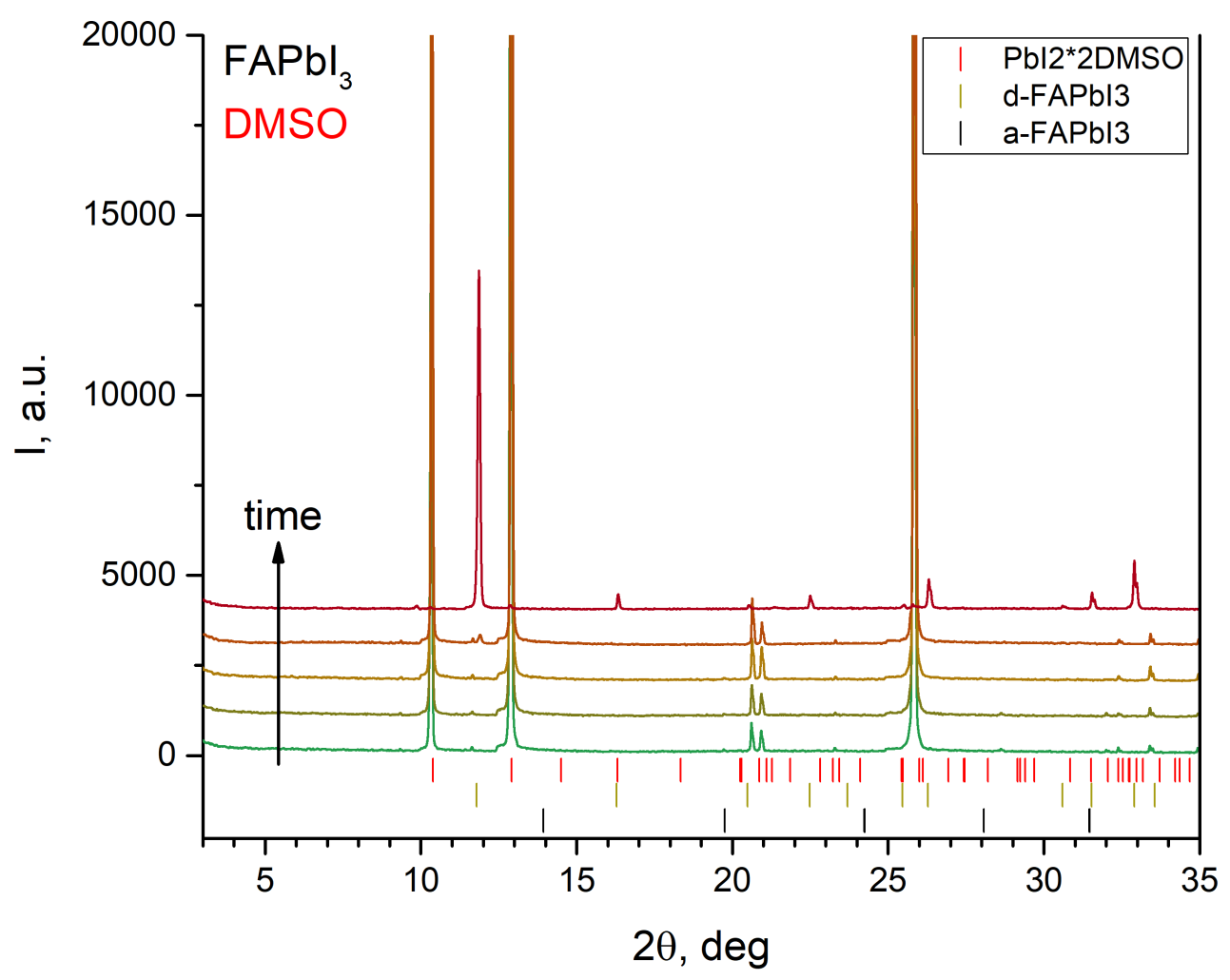

Figure S2. Crystallization of $\mathrm{FAPbl}_{3}$ from DMSO. 


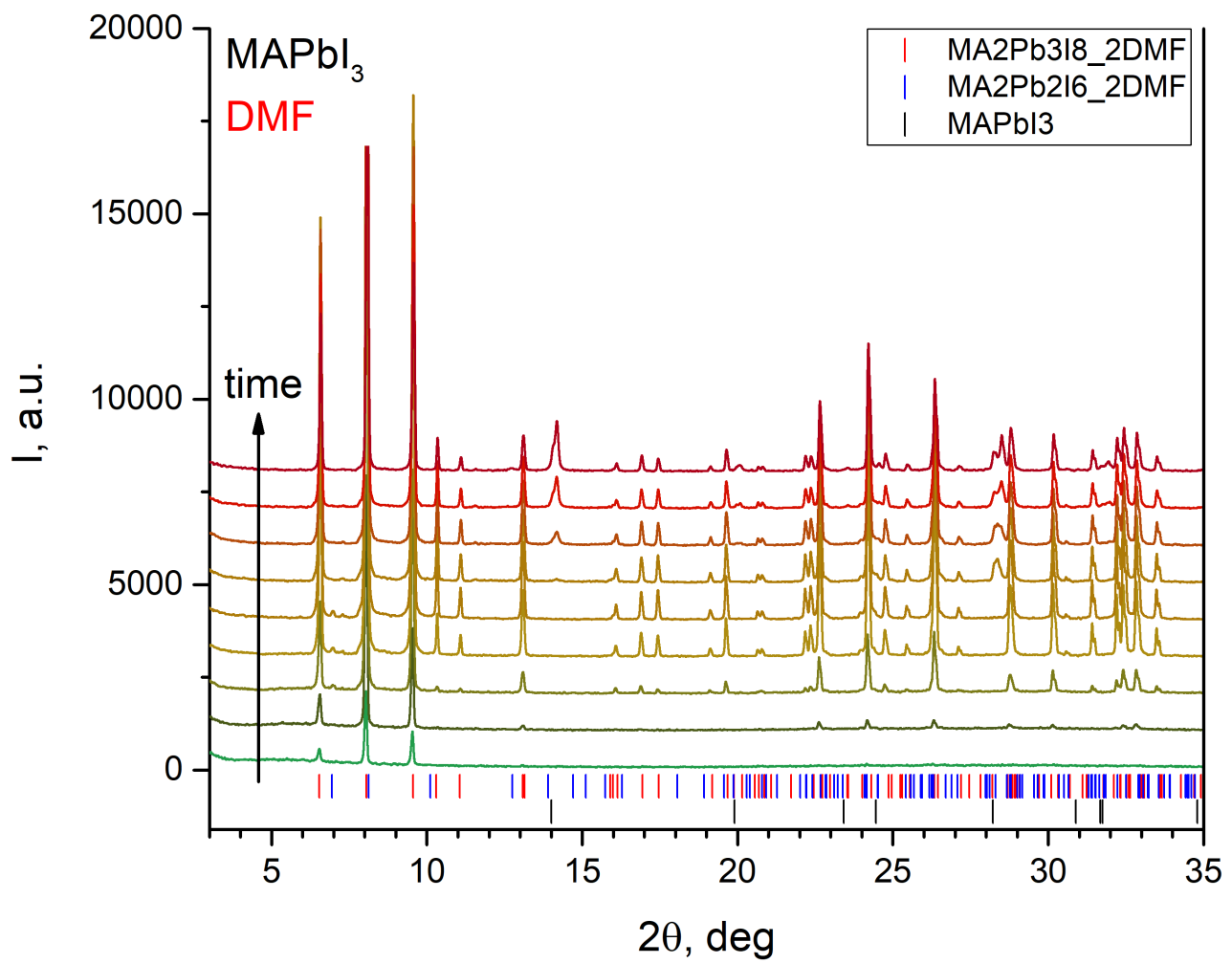

Figure S3. Crystallization of $\mathrm{MAPbl}_{3}$ from DMF.

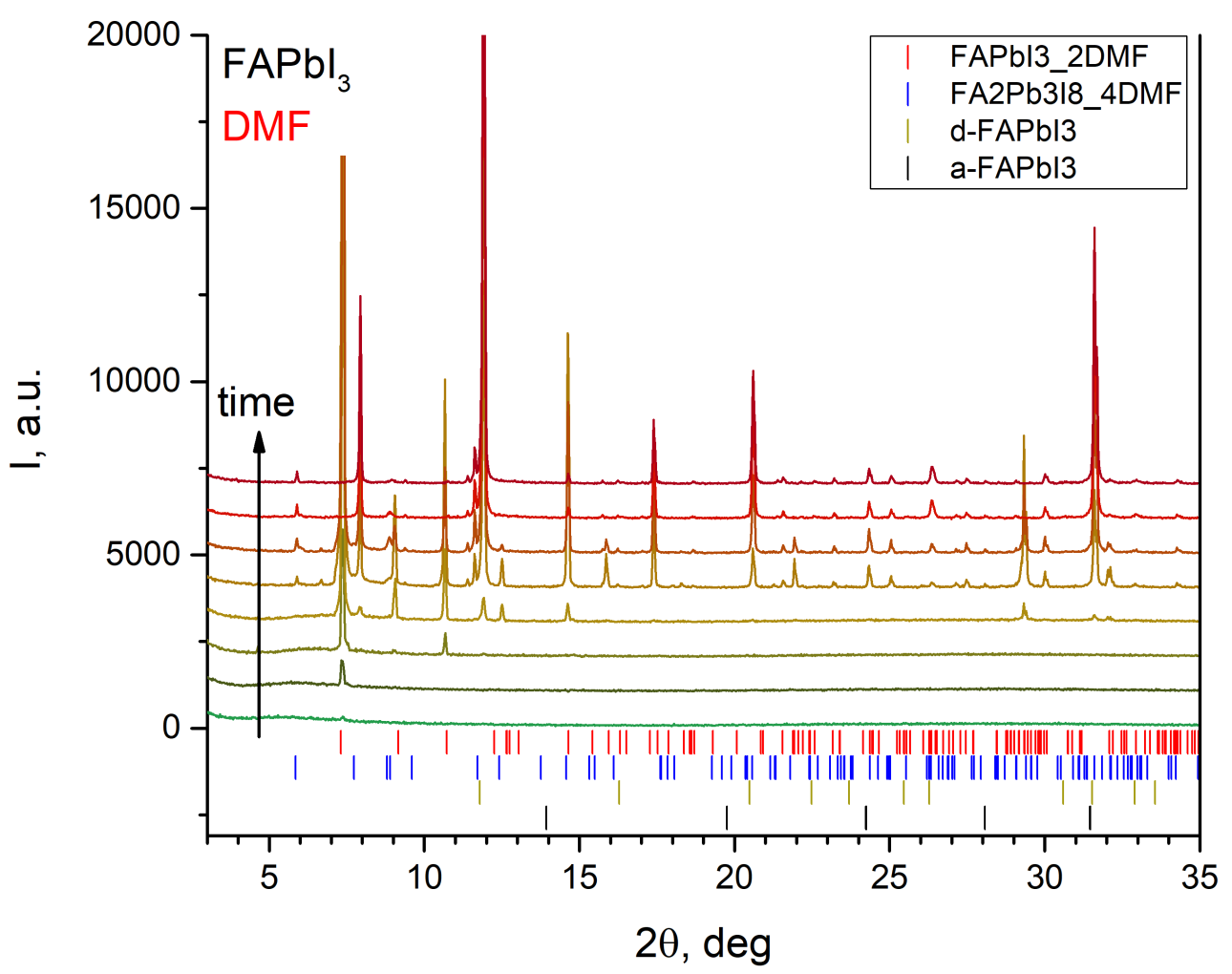

Figure S4. Crystallization of $\mathrm{FAPbl}_{3}$ from DMF. 


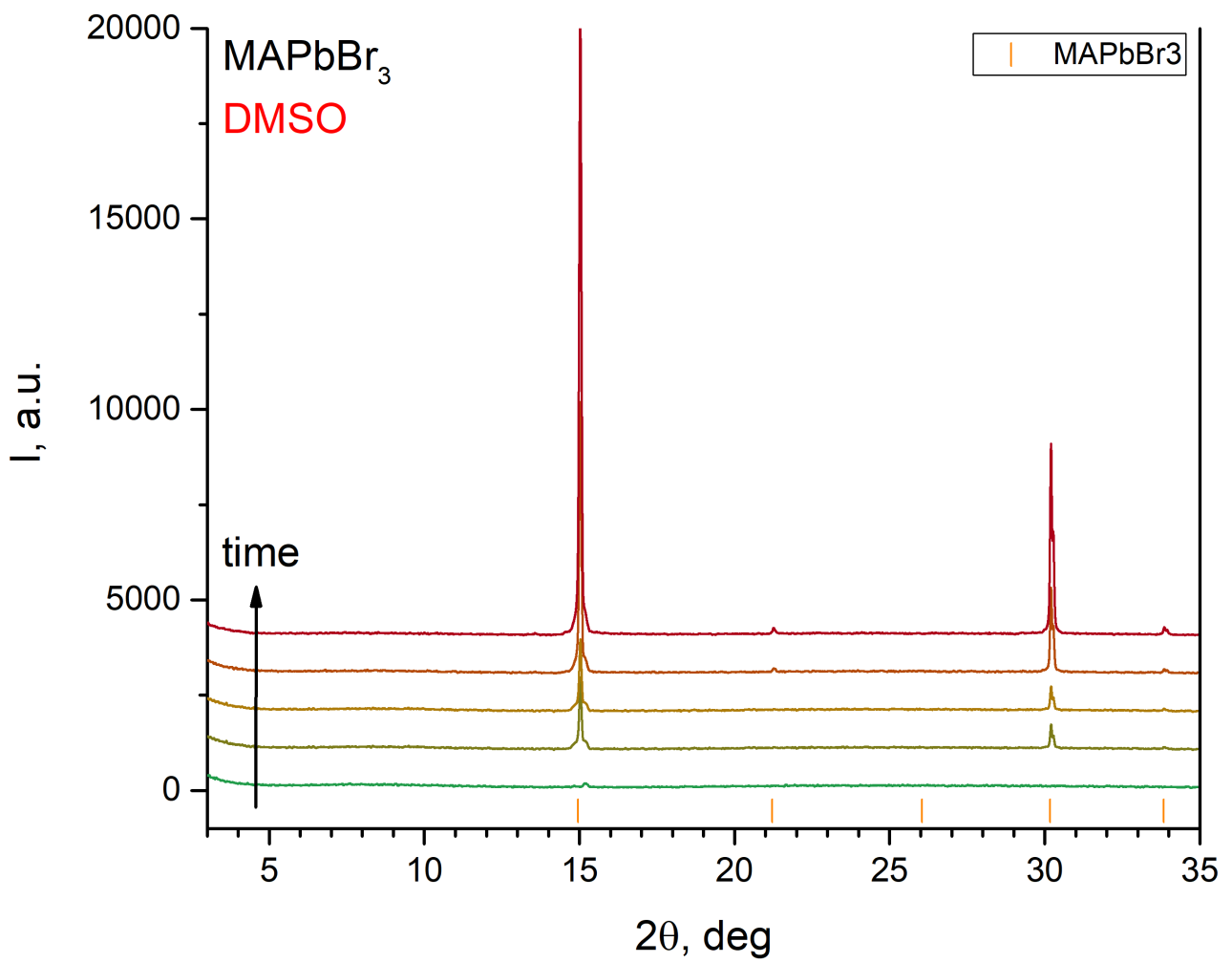

Figure S5. Crystallization of $\mathrm{MAPbBr}_{3}$ from DMSO.

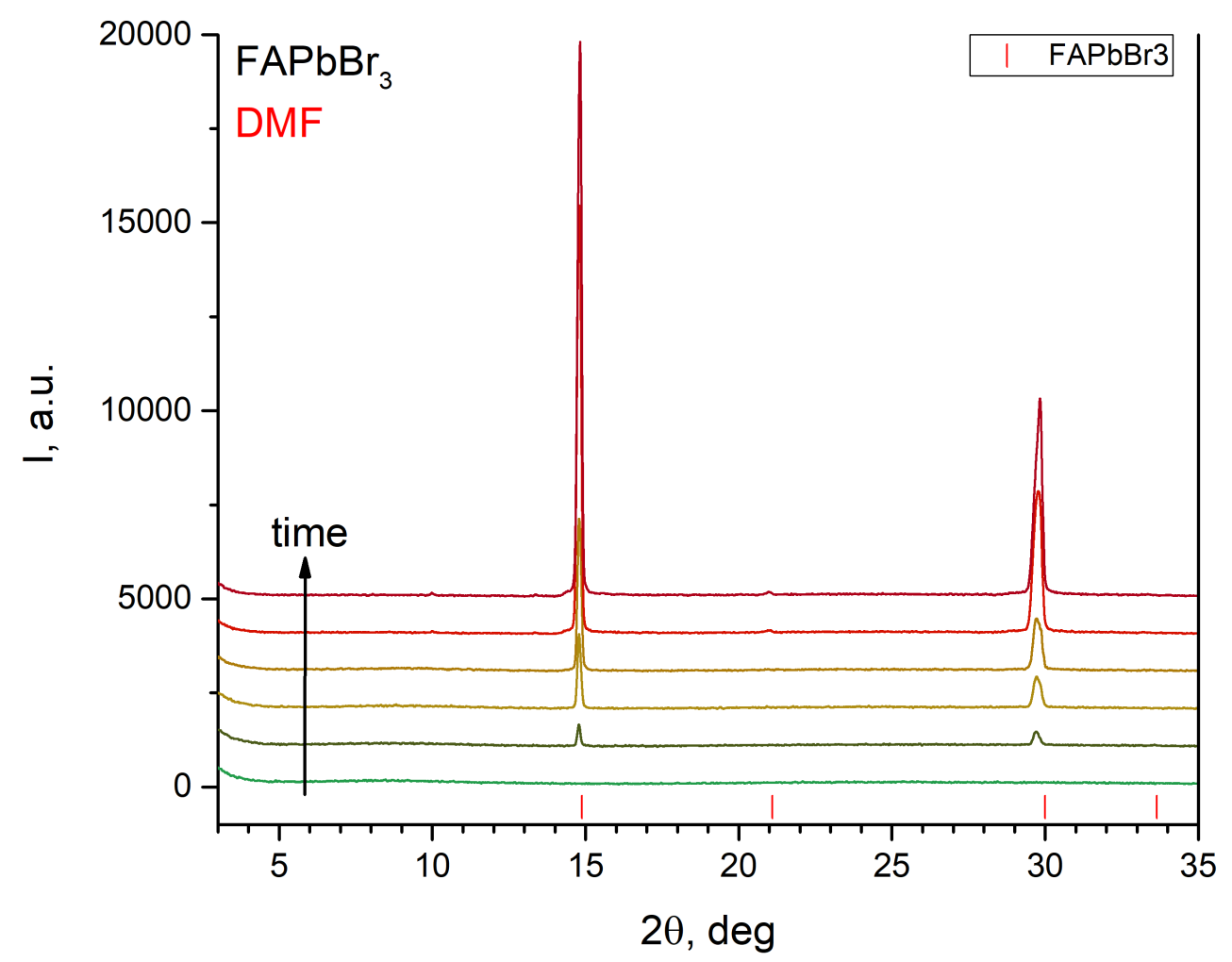

Figure S6. Crystallization of $\mathrm{FAPbBr}_{3}$ from DMSO. 


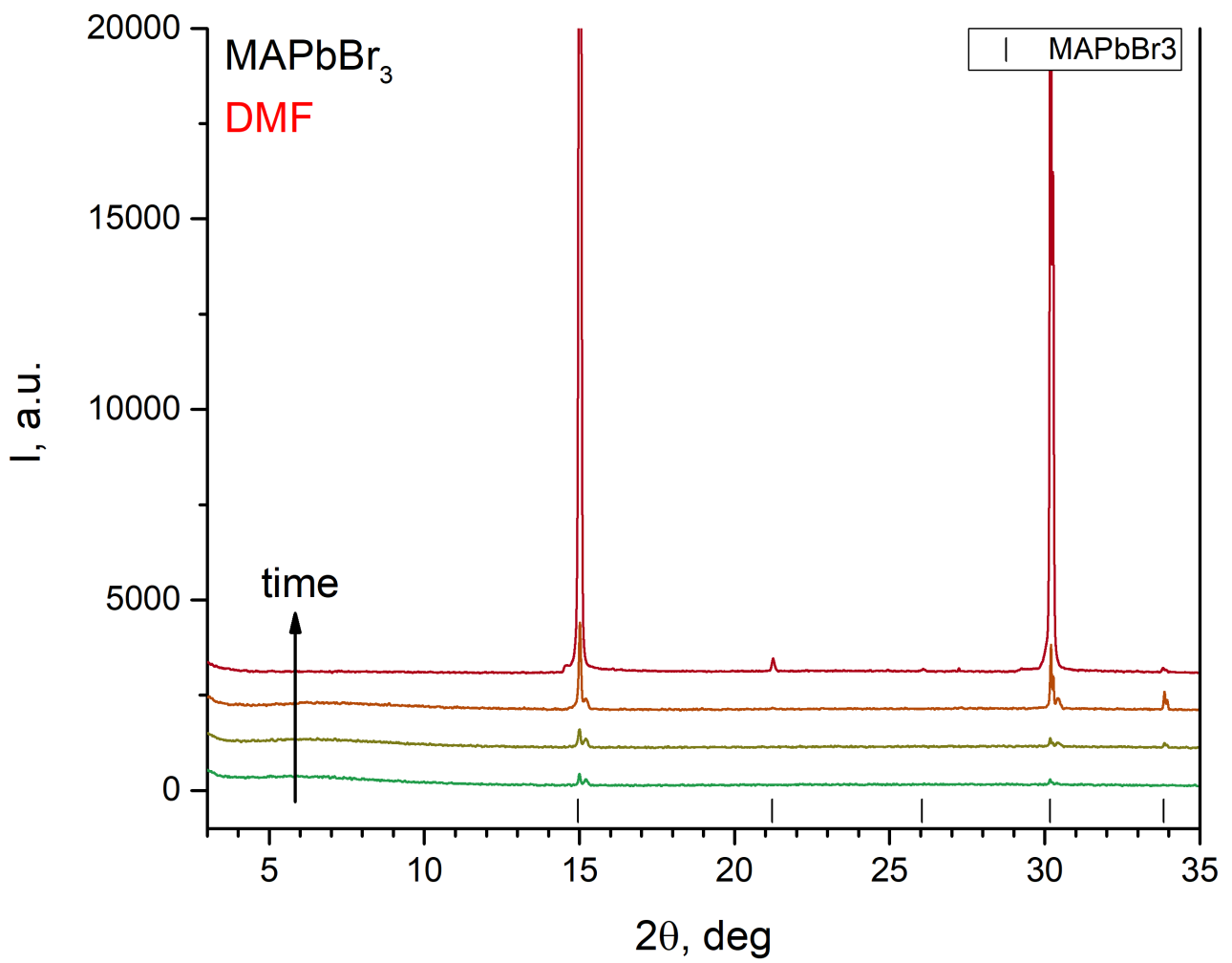

Figure S7. Crystallization of $\mathrm{MAPbBr}_{3}$ from DMF.

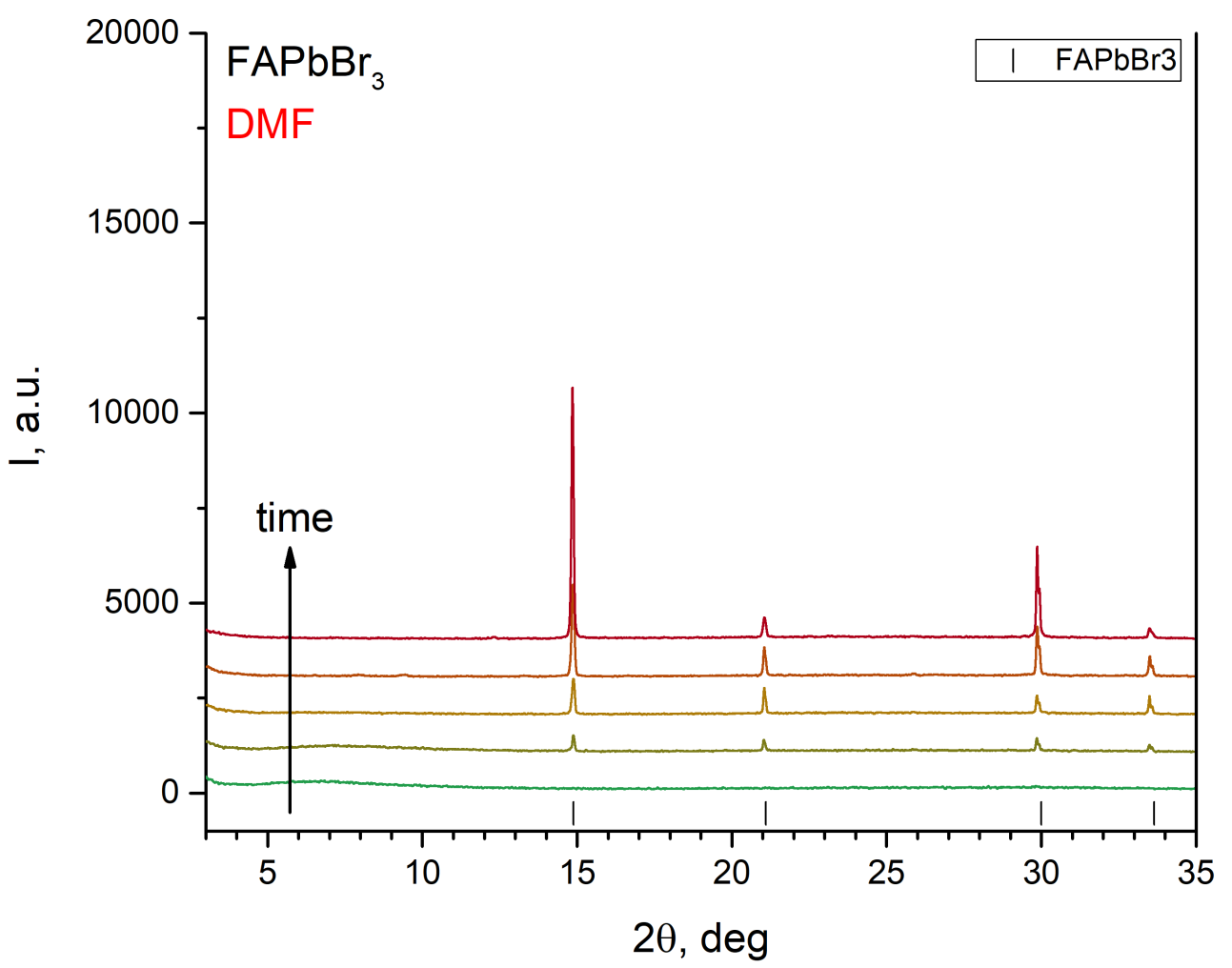

Figure S8. Crystallization of $\mathrm{FAPbBr}_{3}$ from DMF. 


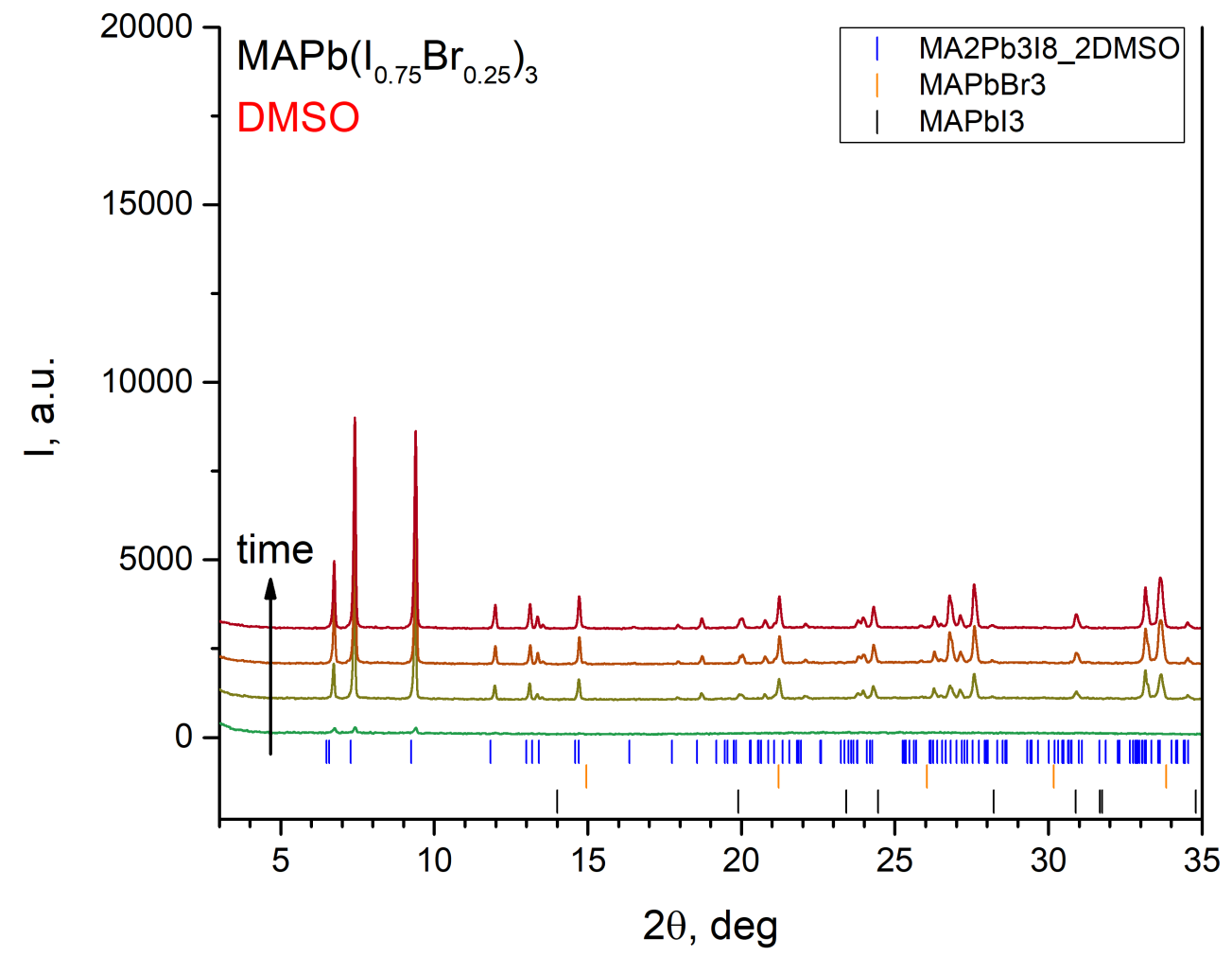

Figure S9. Crystallization of $\mathrm{MAPb}\left(\mathrm{I}_{0.75} \mathrm{Br}_{0.25}\right)_{3}$ from DMSO.

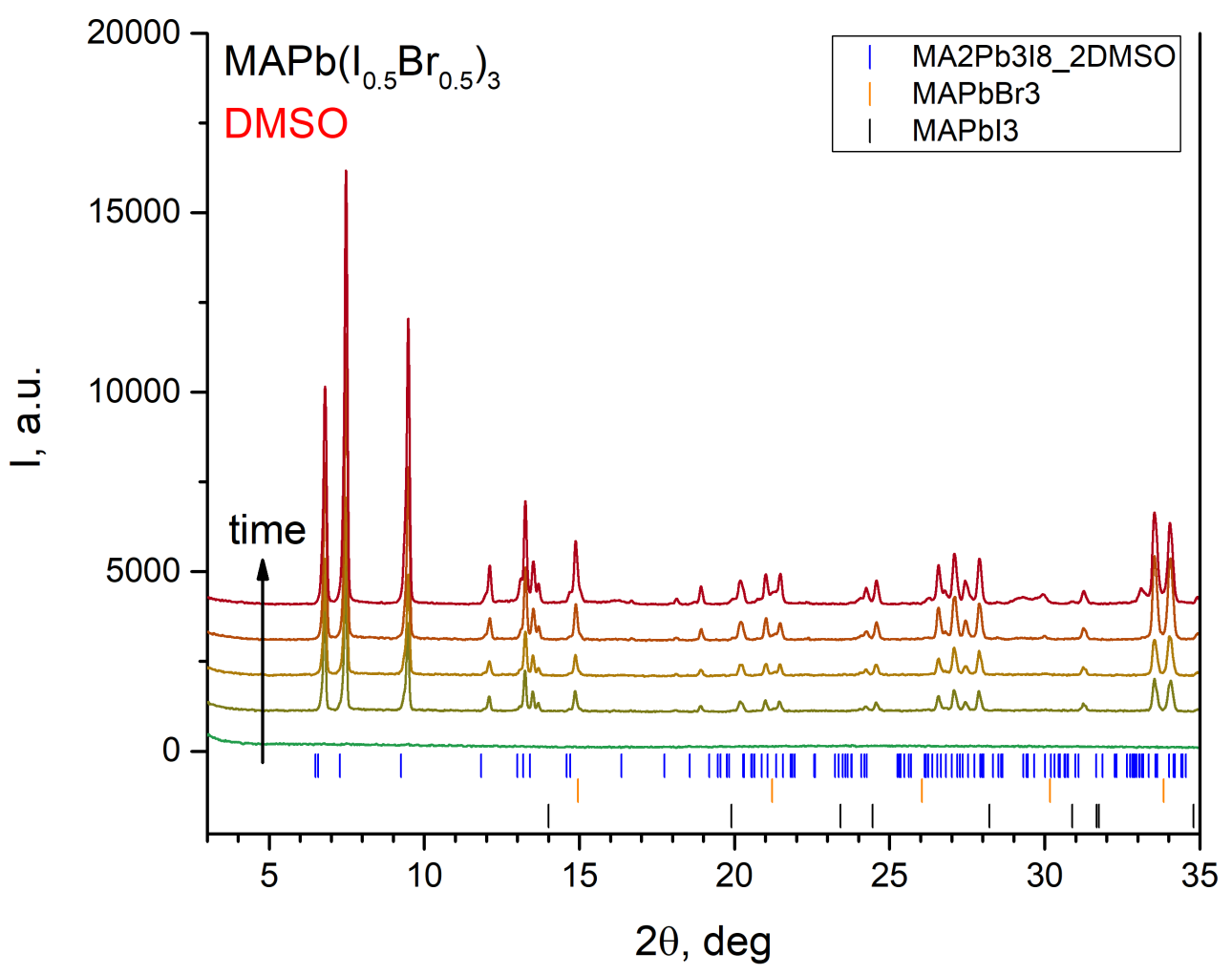

Figure $\mathrm{S10}$. Crystallization of $\mathrm{MAPb}\left(\mathrm{l}_{0.5} \mathrm{Br}_{0.5}\right)_{3}$ from DMSO. 


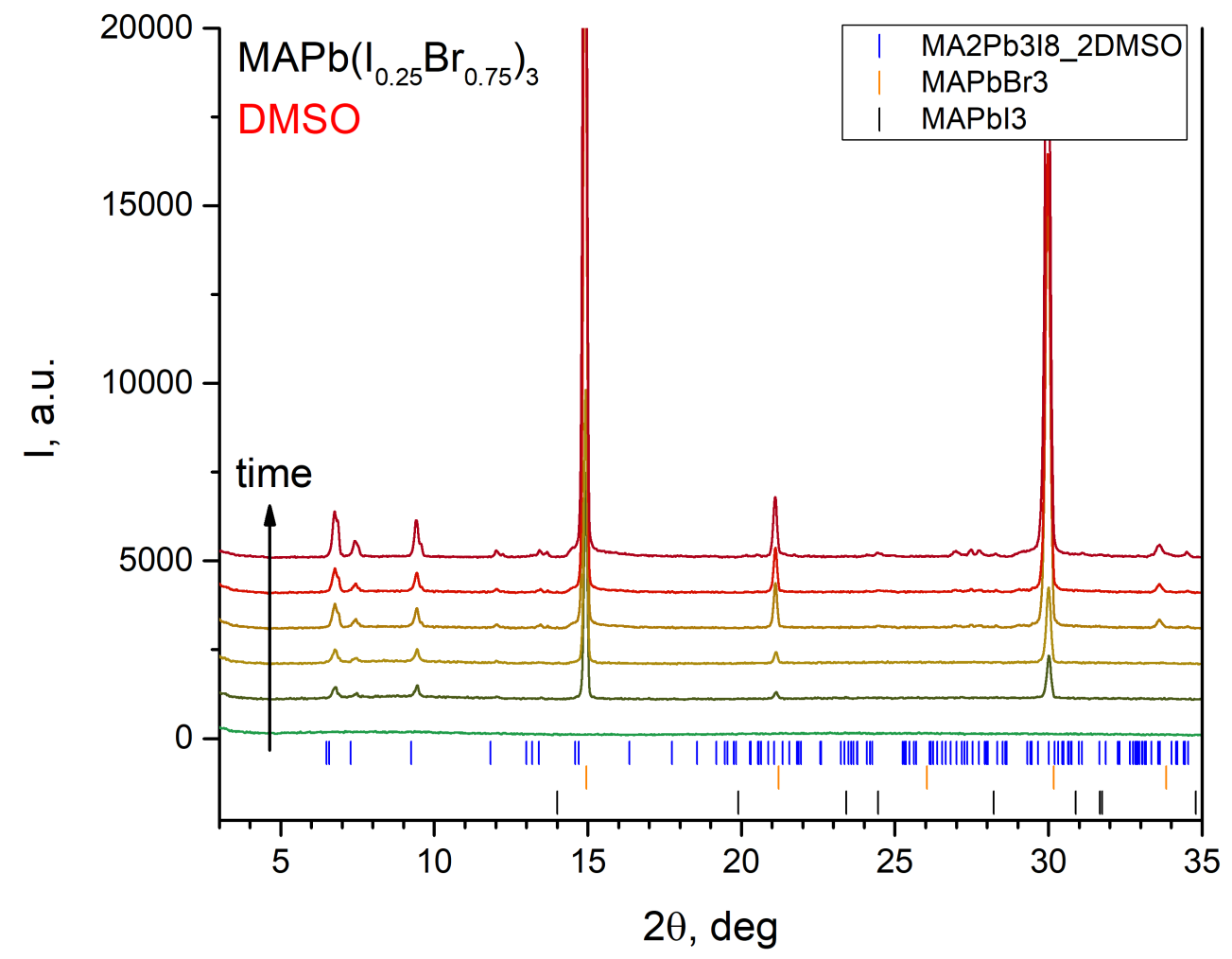

Figure S11. Crystallization of $\mathrm{MAPb}\left(\mathrm{I}_{0.25} \mathrm{Br} \mathrm{r}_{0.75}\right)_{3}$ from DMSO.
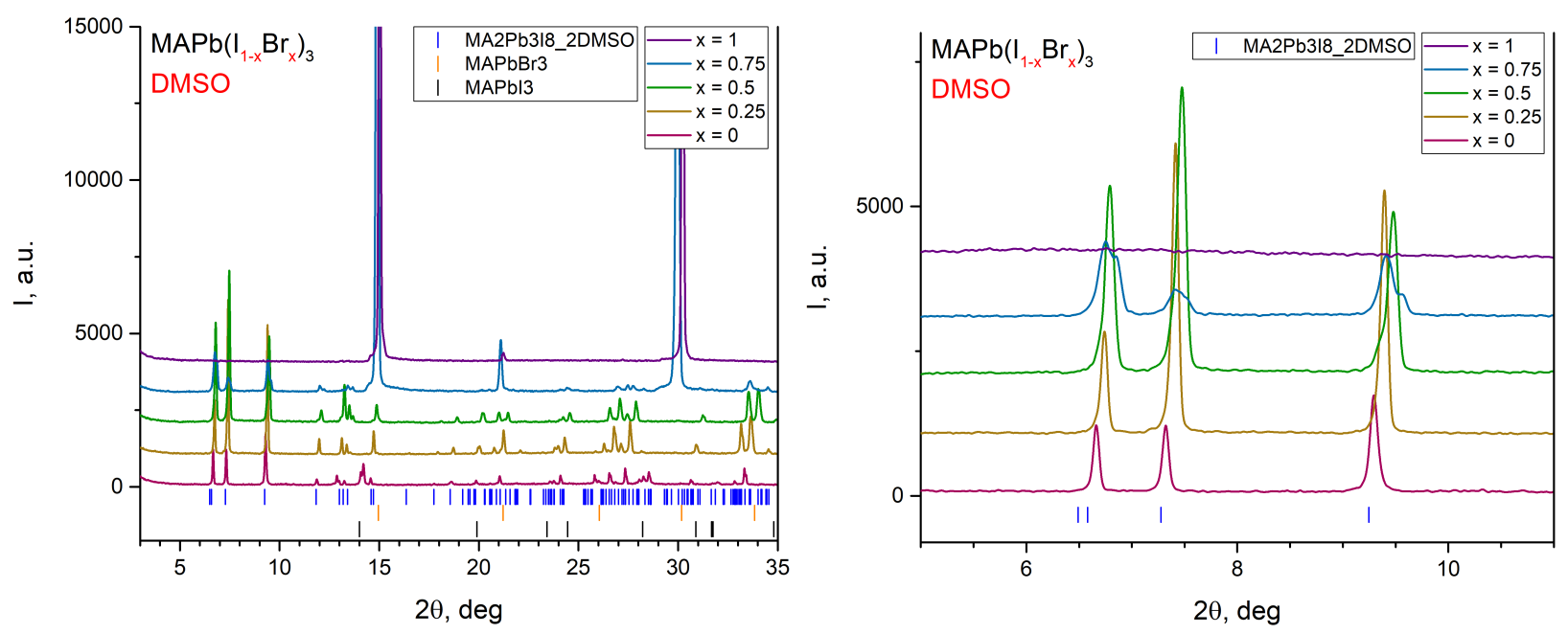

Figure S12. Crystallization of $\mathrm{MAPb}\left(1_{1-x} \mathrm{Br}_{\mathrm{x}}\right)_{3}$ from DMSO. 


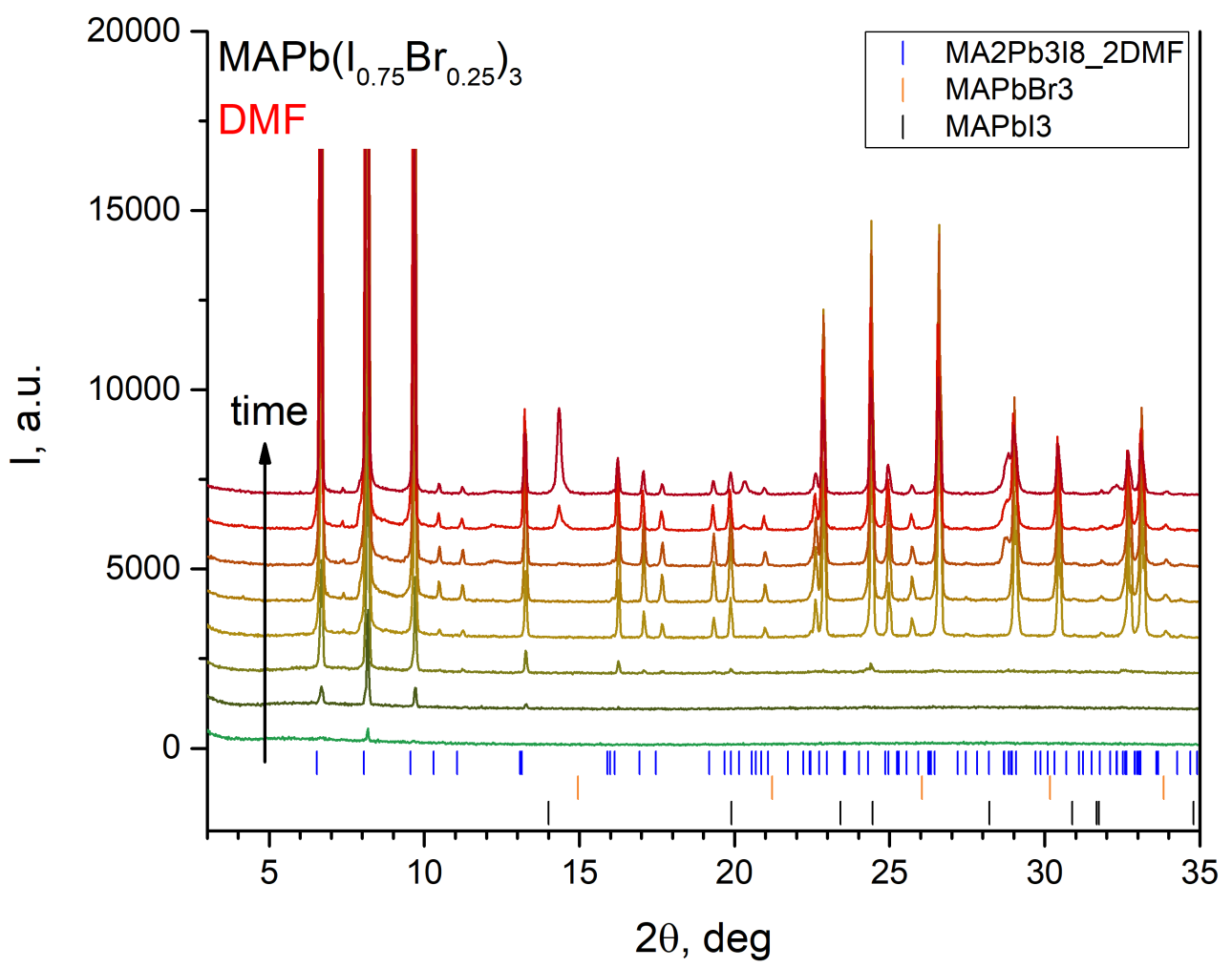

Figure S13. Crystallization of $\mathrm{MAPb}\left(\mathrm{I}_{0.75} \mathrm{Br}_{0.25}\right)_{3}$ from DMF.

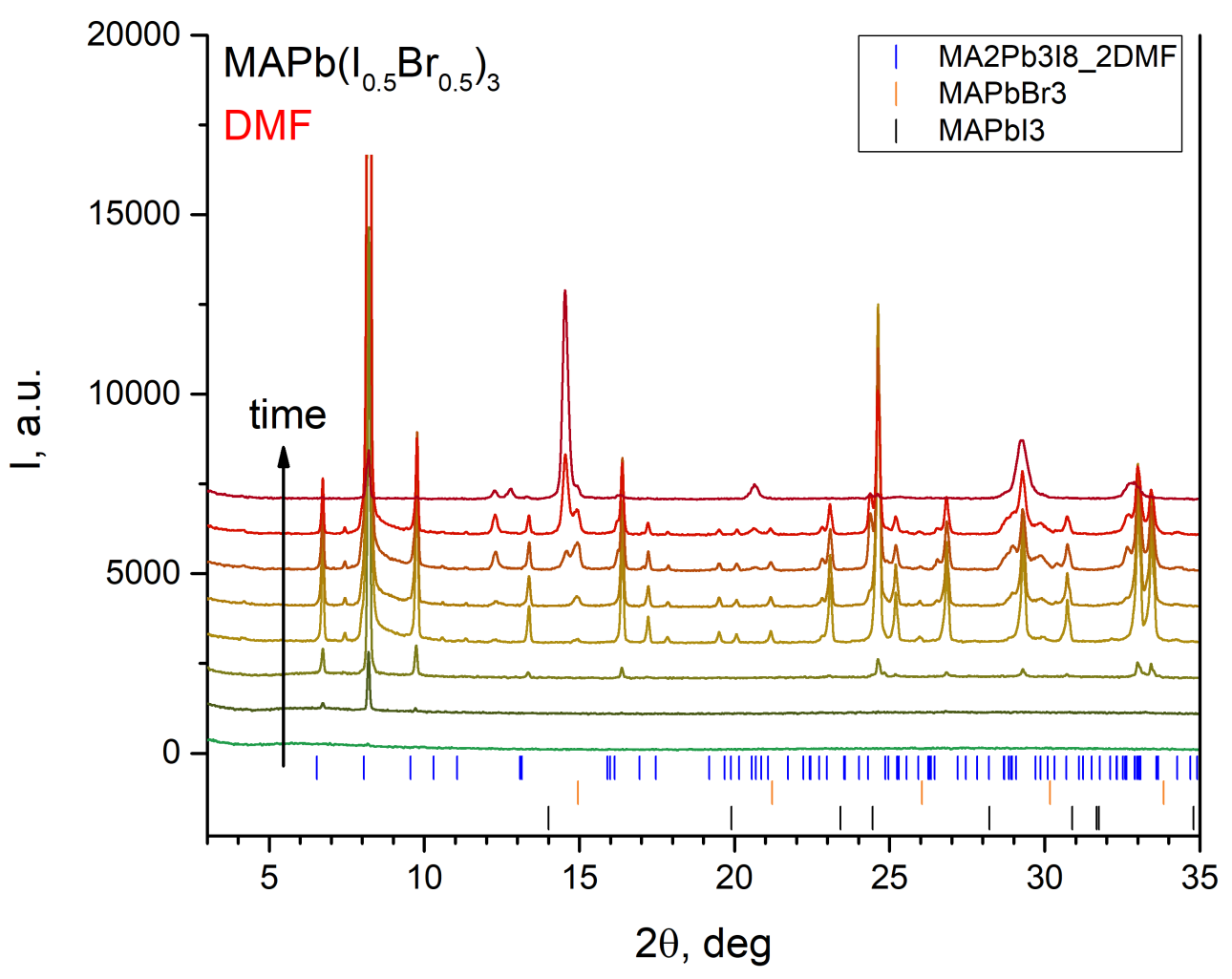

Figure S14. Crystallization of $\mathrm{MAPb}\left(\mathrm{I}_{0.5} \mathrm{Br}_{0.5}\right)_{3}$ from DMF. 


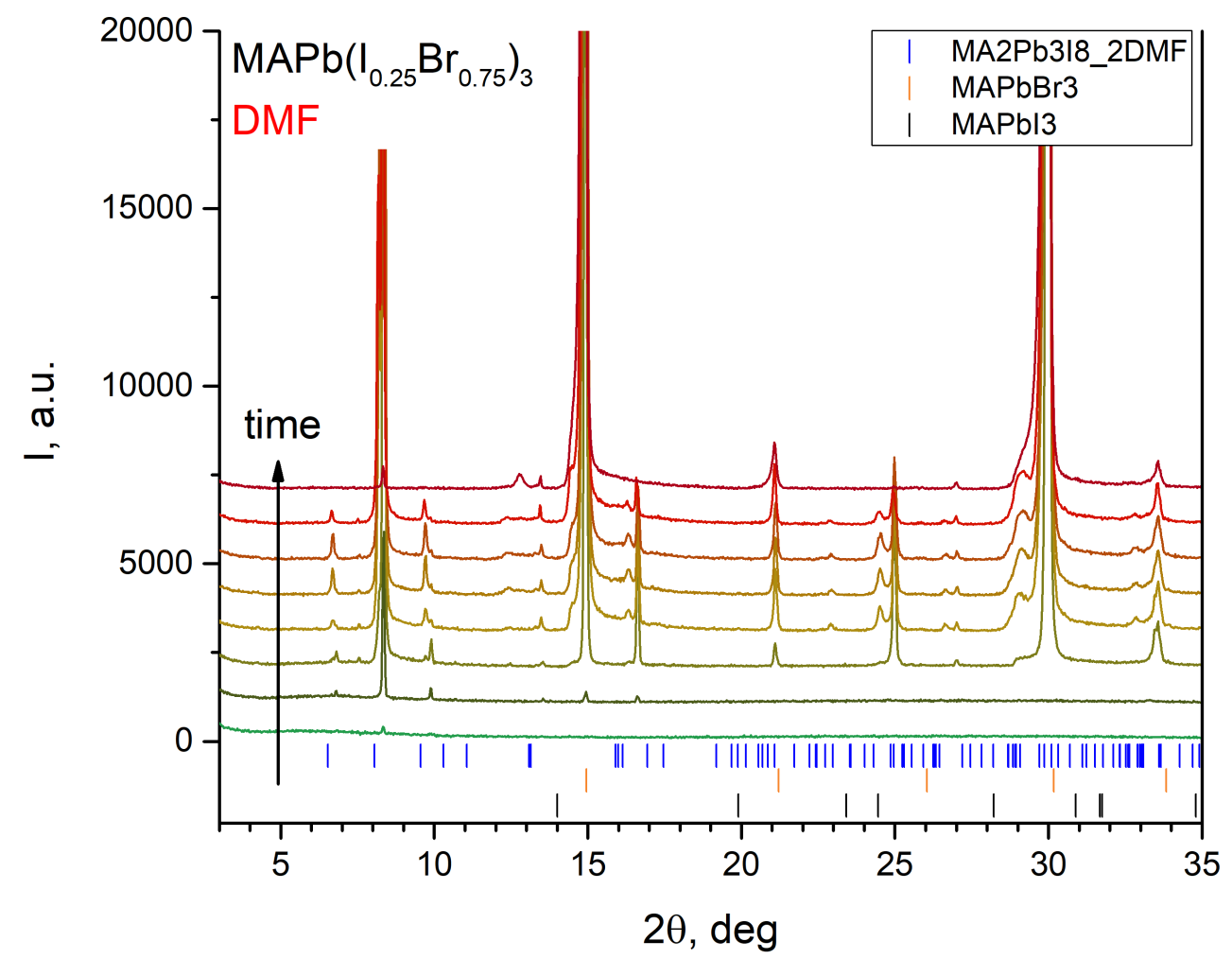

Figure $\mathrm{S15}$. Crystallization of $\mathrm{MAPb}\left(\mathrm{I}_{0.25} \mathrm{Br} \mathrm{r}_{0.75}\right)_{3}$ from $\mathrm{DMF}$.
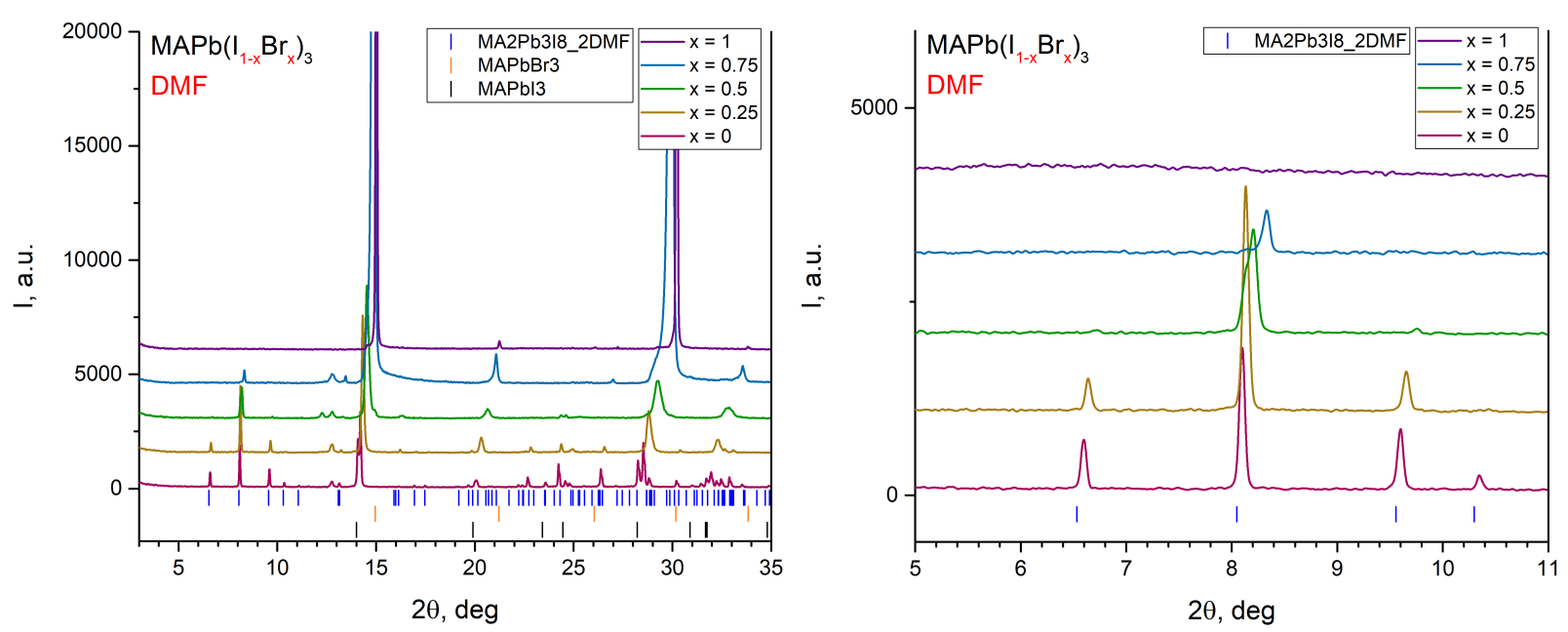

Figure S16. Crystallization of $\mathrm{MAPb}\left(\mathrm{I}_{1-\mathrm{x}} \mathrm{Br} \mathrm{r}_{\mathrm{x}}\right)_{3}$ from DMF. 


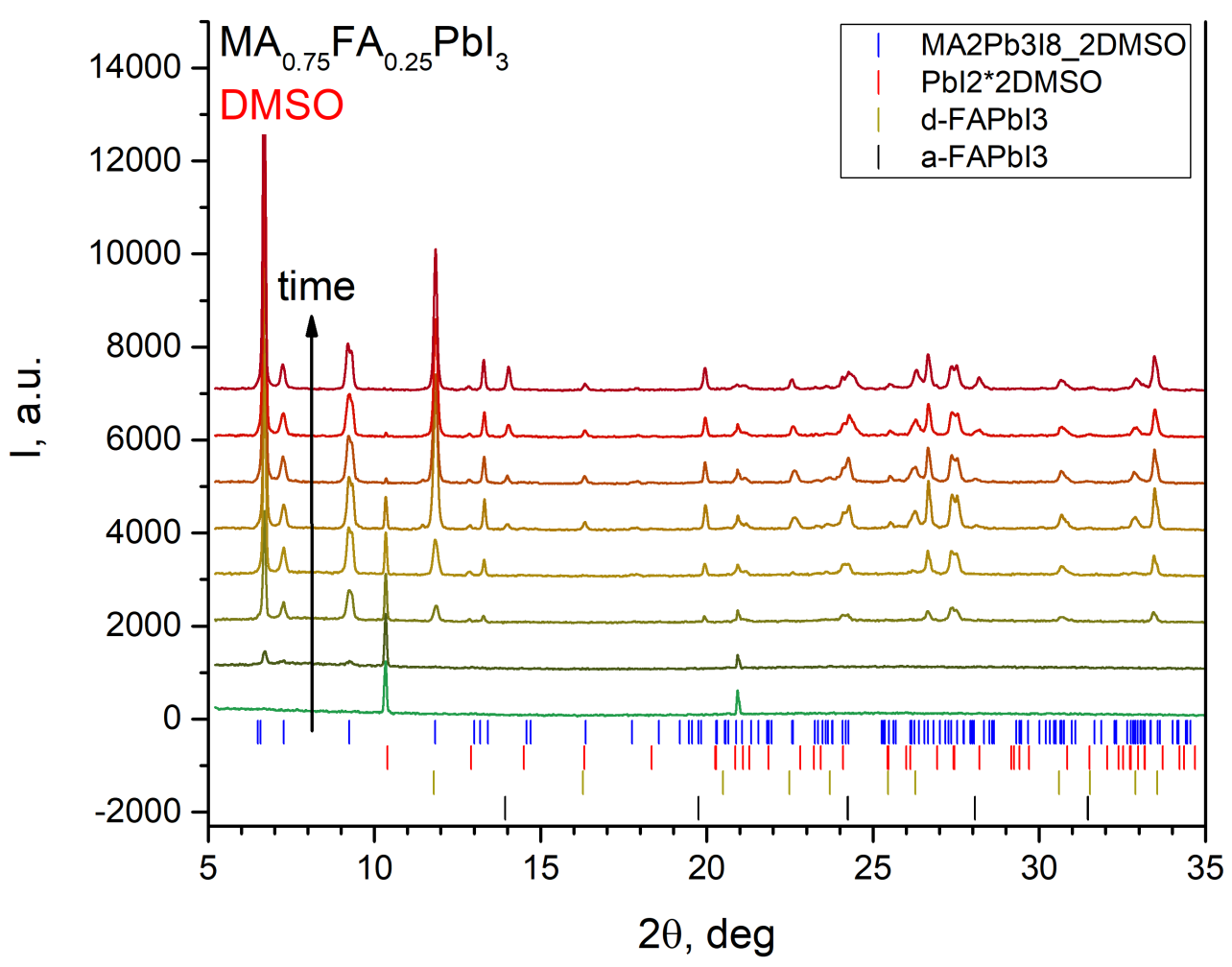

Figure S17. Crystallization of $\mathrm{MA}_{0.75} \mathrm{FA}_{0.25} \mathrm{Pbl}_{3}$ from DMSO.

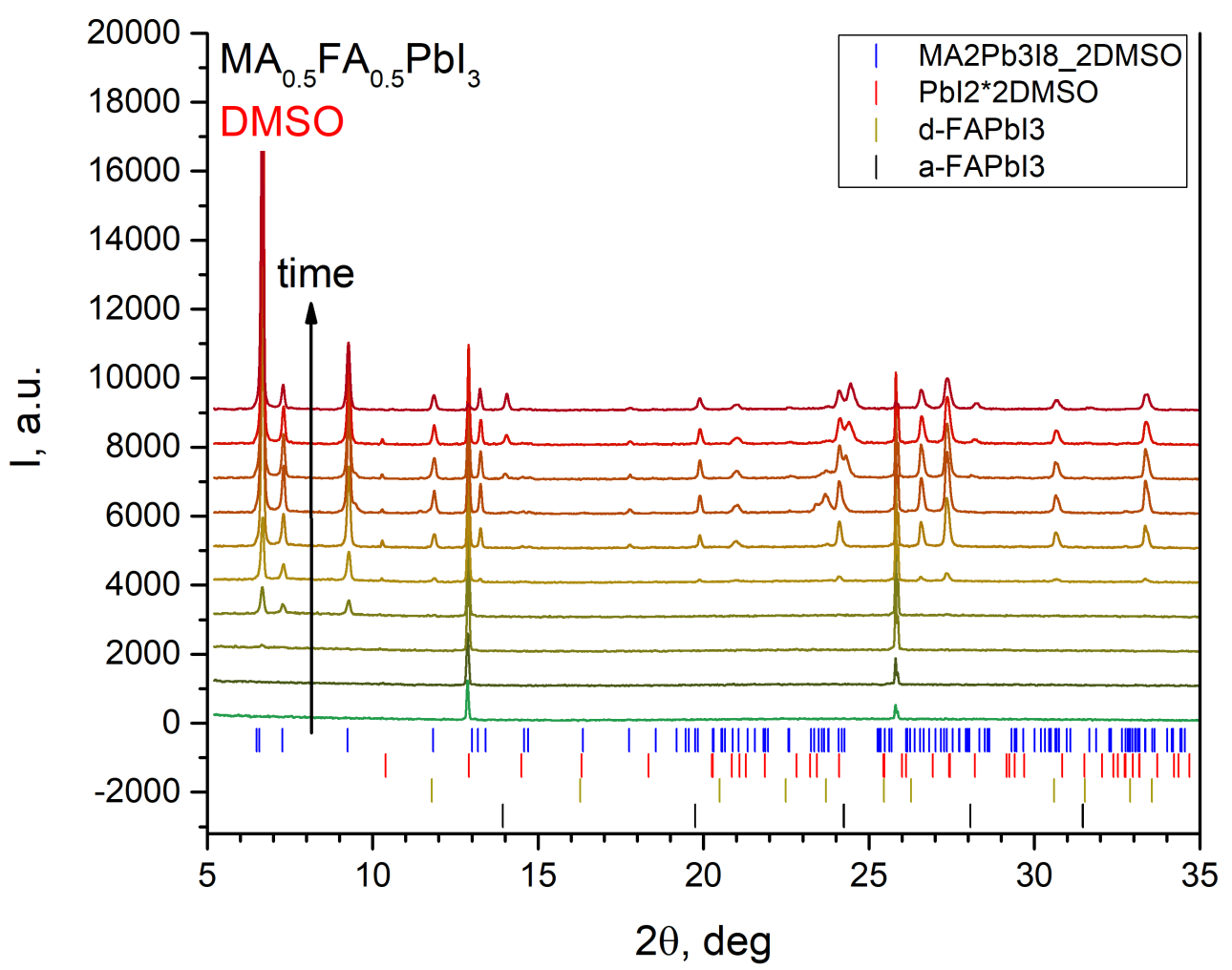

Figure S18. Crystallization of $\mathrm{MA}_{0.5} \mathrm{FA}_{0.5} \mathrm{Pbl}_{3}$ from DMSO. 


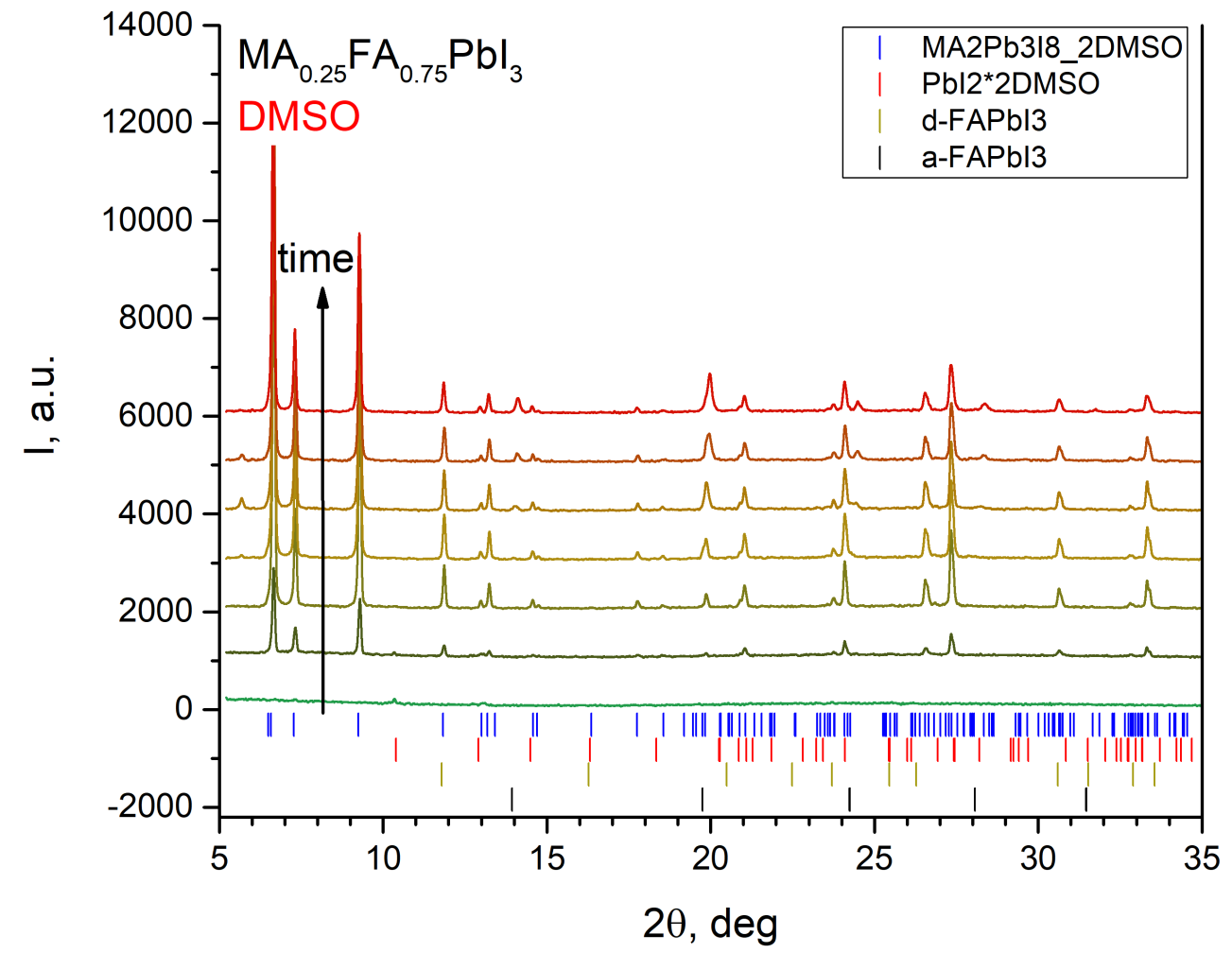

Figure S19. Crystallization of $\mathrm{MA}_{0.25} \mathrm{FA}_{0.75} \mathrm{Pbl}_{3}$ from DMSO.
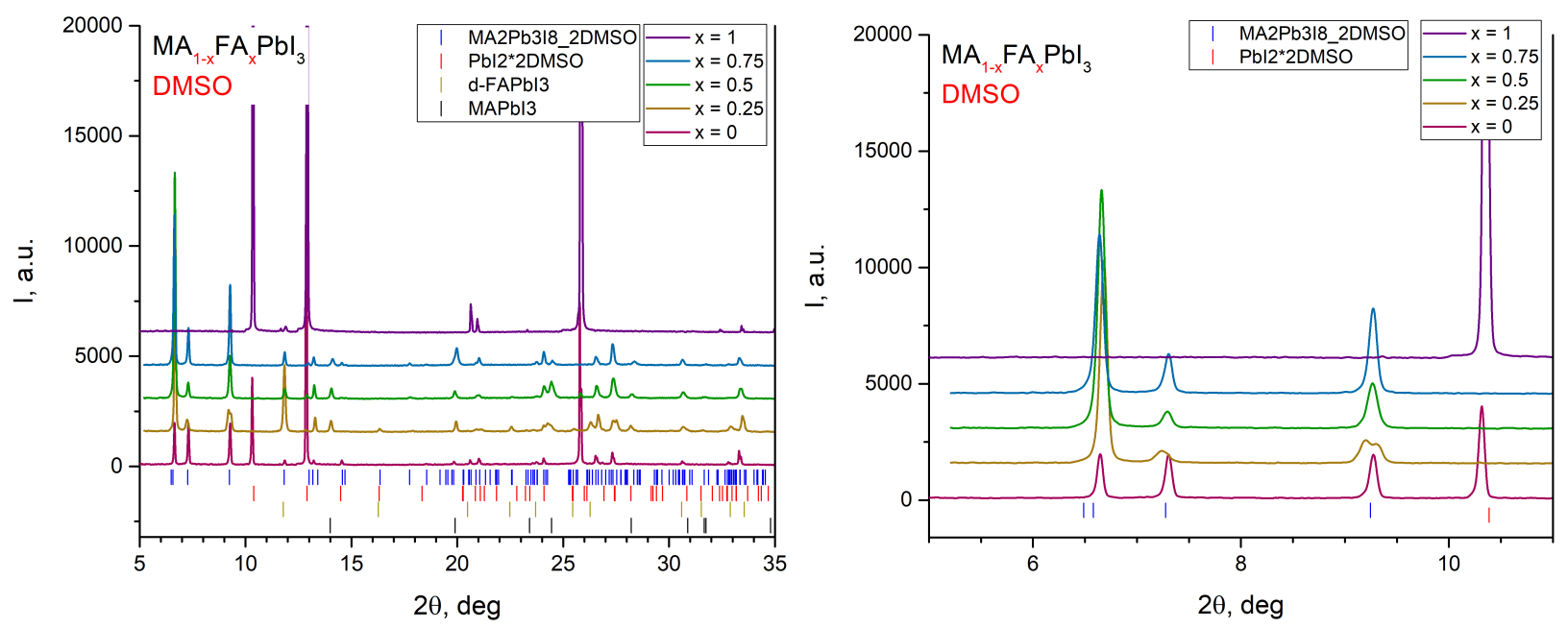

Figure S20. Crystallization of $\mathrm{MA}_{1-x} \mathrm{FA}_{x} \mathrm{Pbl}_{3}$ from DMSO. 


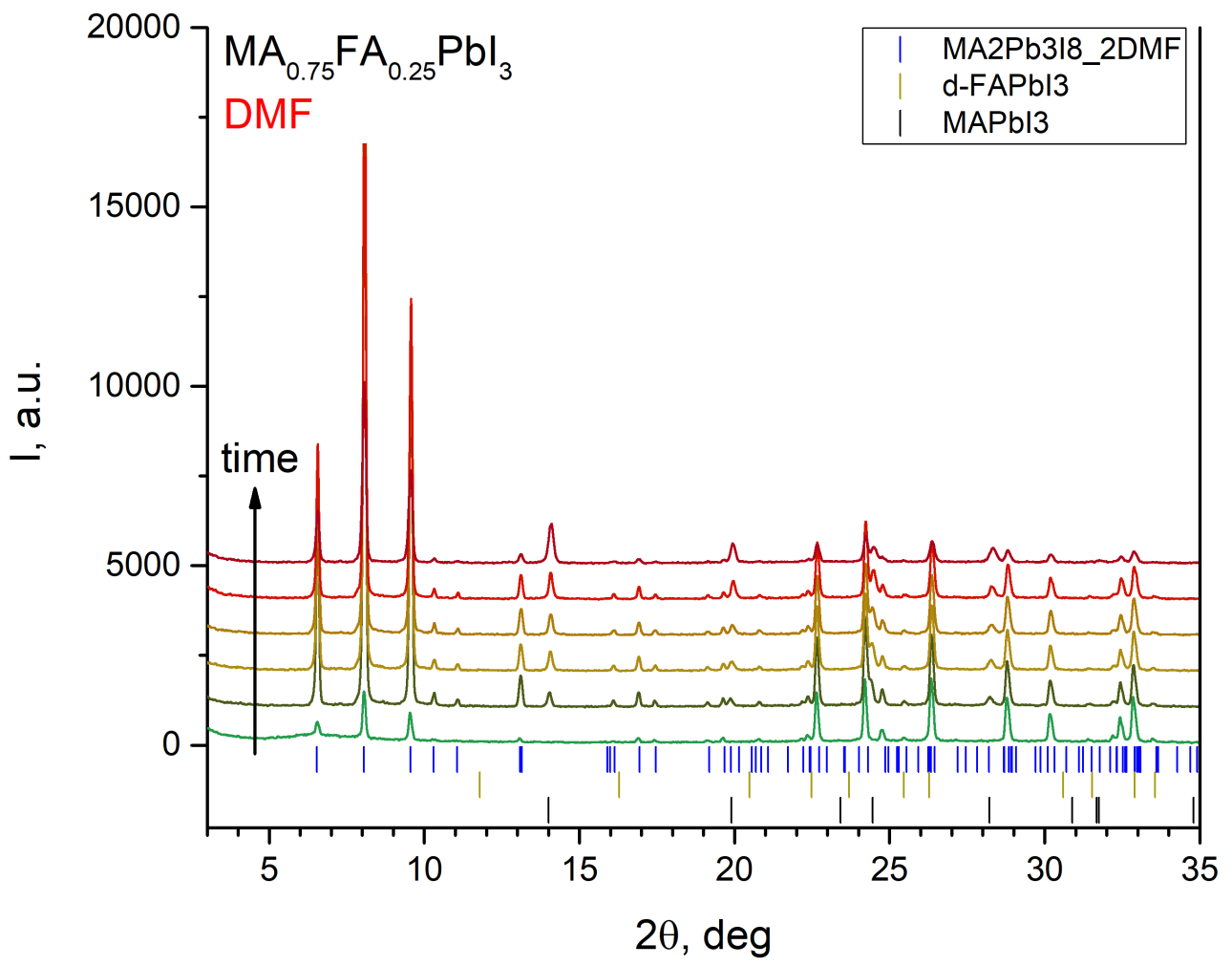

Figure S21. Crystallization of $\mathrm{MA}_{0.75} \mathrm{FA}_{0.25} \mathrm{Pbl}_{3}$ from DMF.

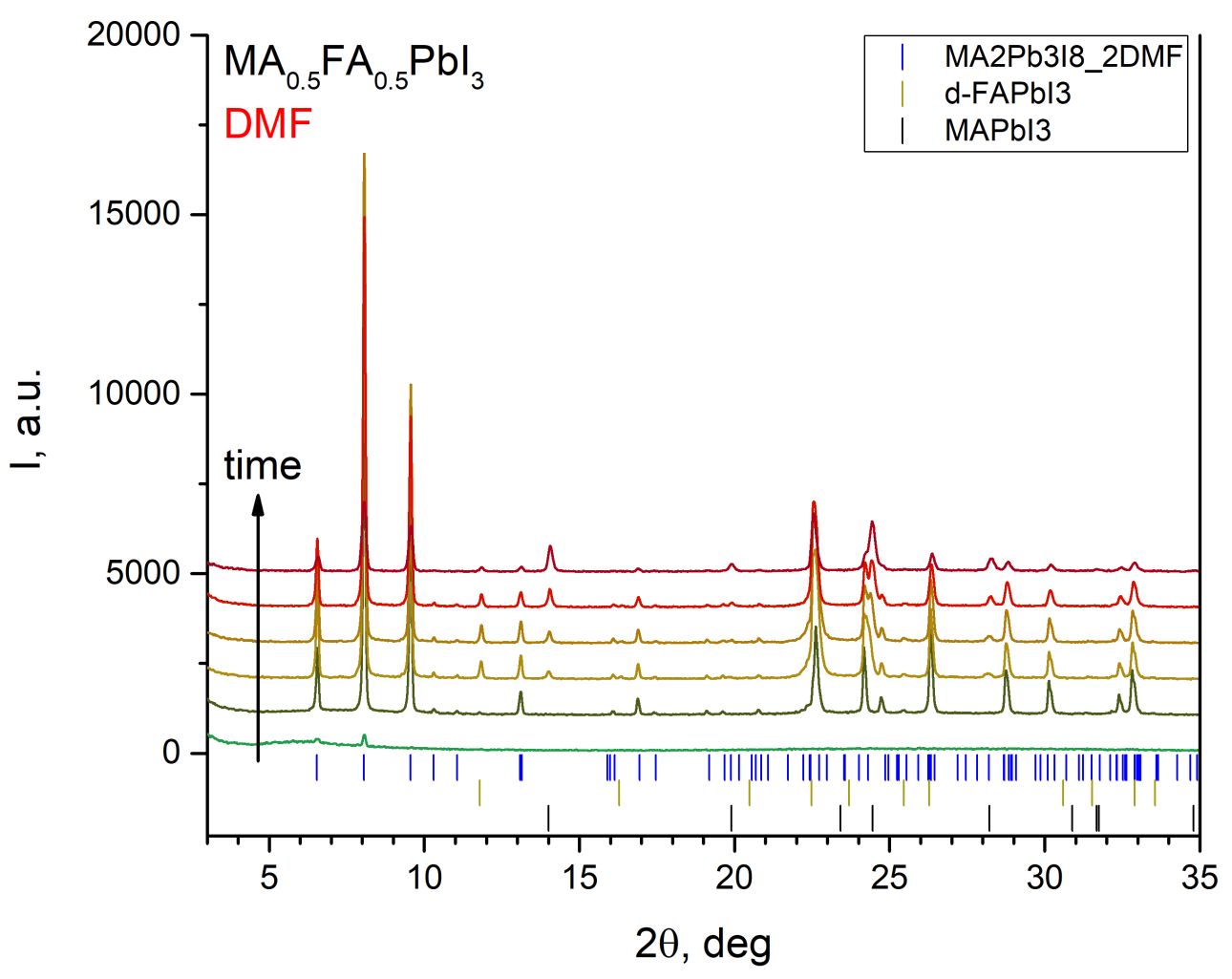

Figure S22. Crystallization of $\mathrm{MA}_{0.5} \mathrm{FA}_{0.5} \mathrm{Pbl}_{3}$ from $\mathrm{DMF}$. 


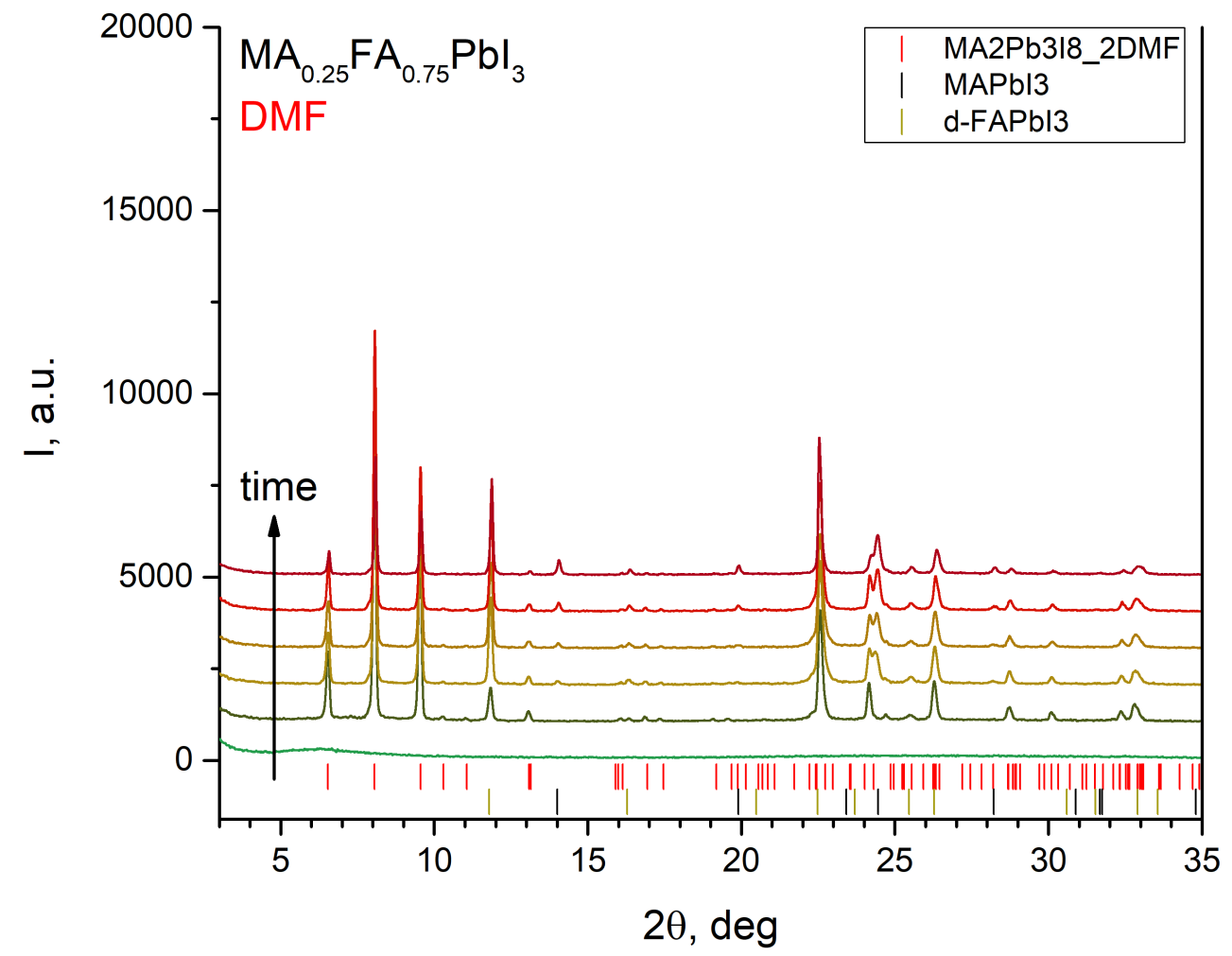

Figure S23. Crystallization of $\mathrm{MA}_{0.25} \mathrm{FA}_{0.75} \mathrm{Pbl}_{3}$ from DMF.
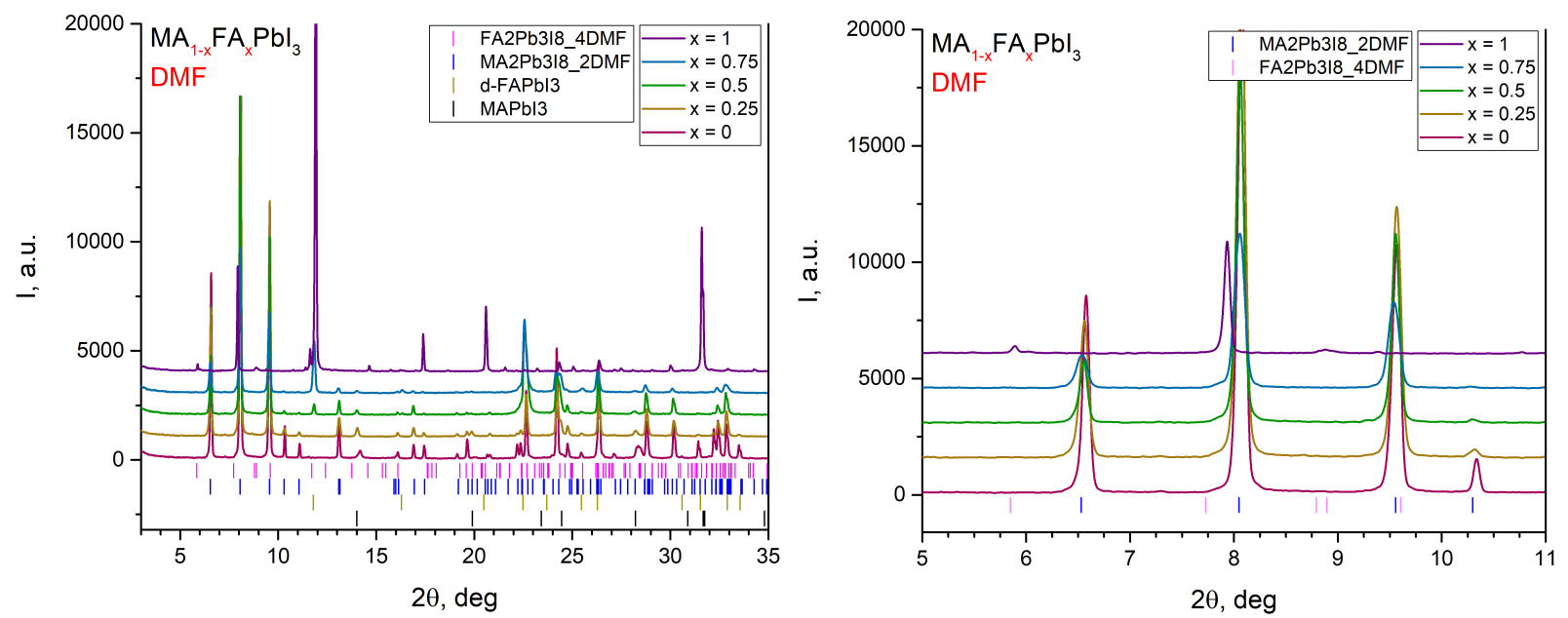

Figure S24. Crystallization of $\mathrm{MA}_{1-\mathrm{x}} \mathrm{FA}_{\mathrm{x}} \mathrm{Pbl}_{3}$ from $\mathrm{DMF}$. 


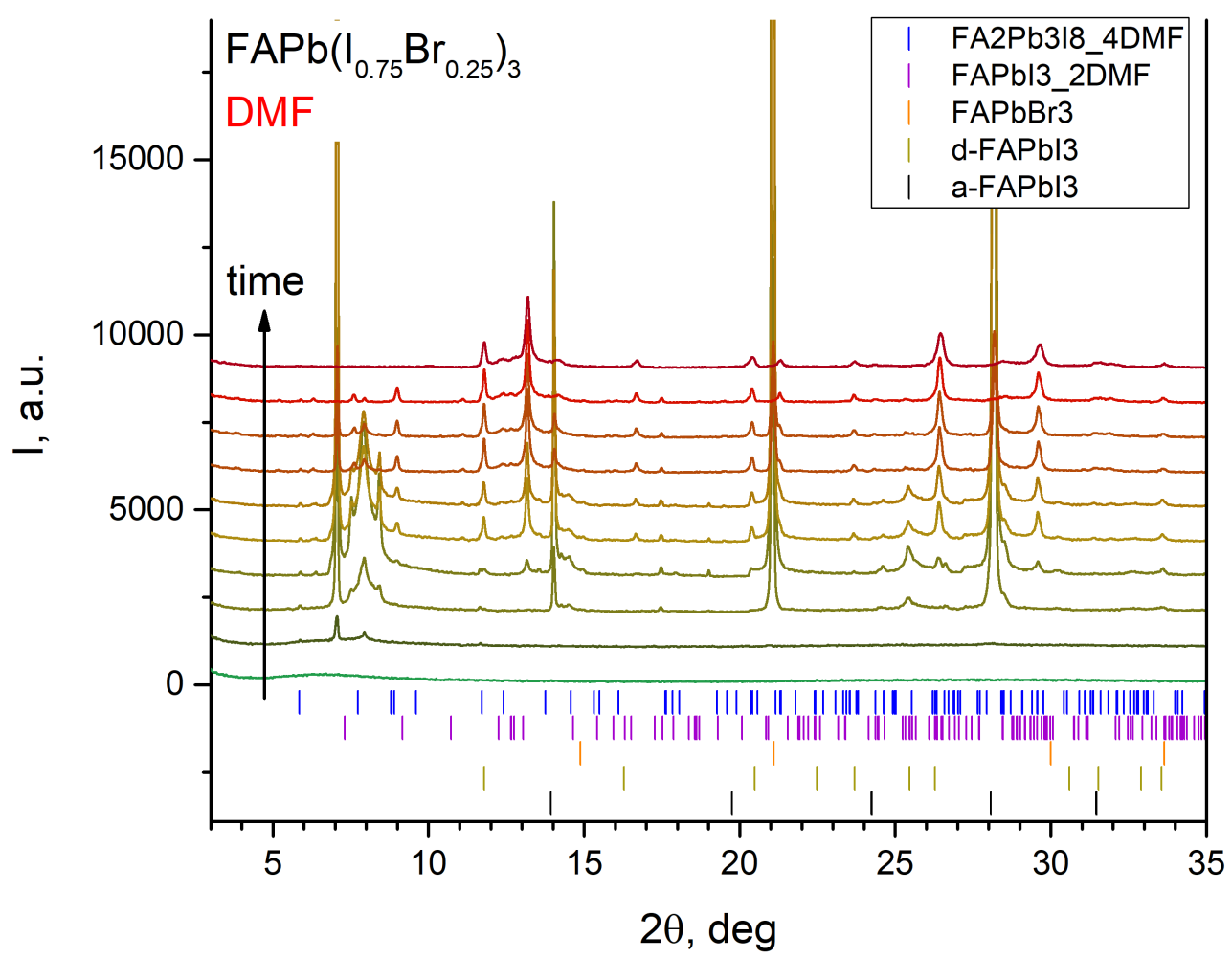

Figure S25. Crystallization of $\mathrm{FAPb}\left(\mathrm{I}_{0.75} \mathrm{Br} \mathrm{O}_{.25}\right)_{3}$ from DMF.

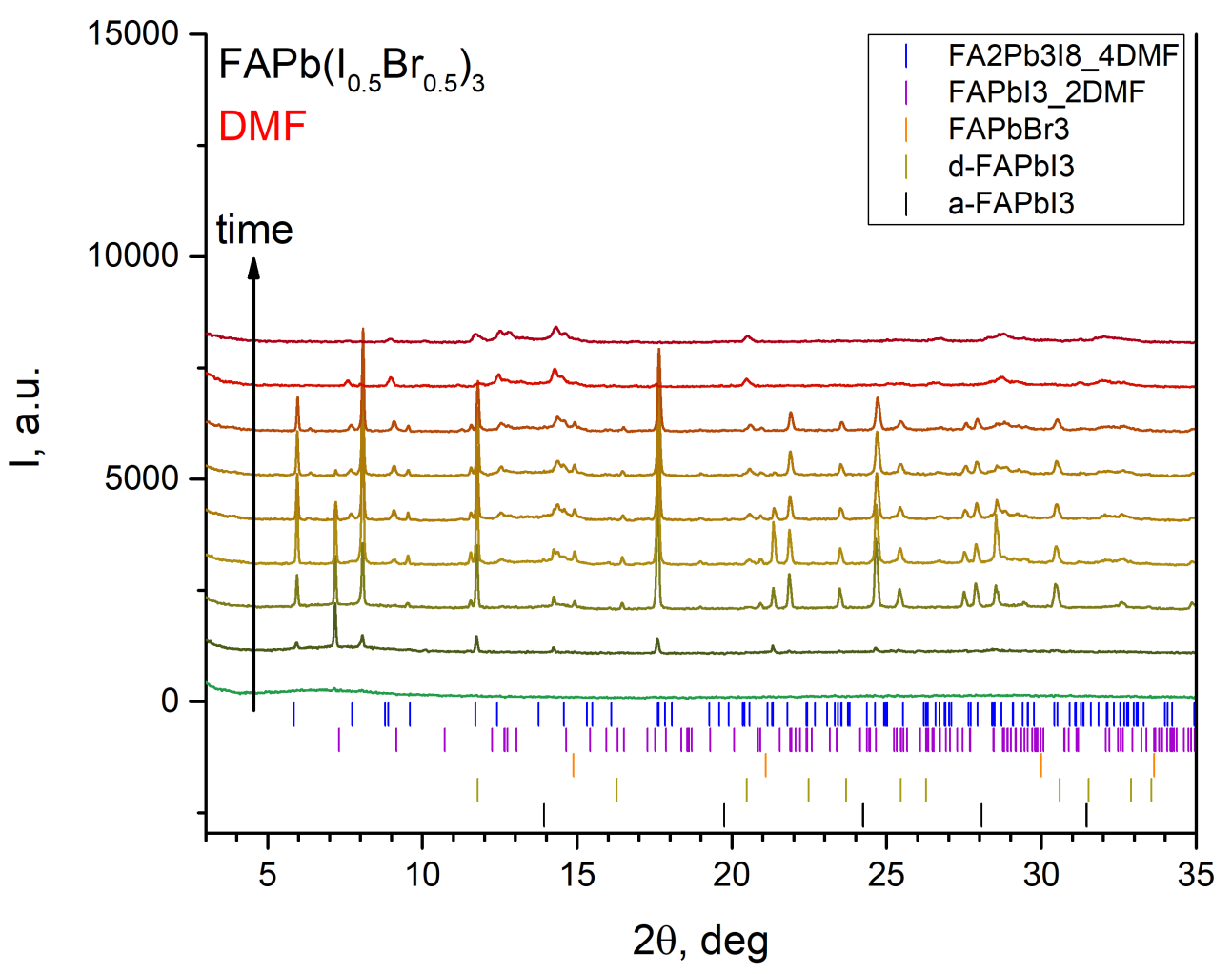

Figure S26. Crystallization of $\mathrm{FAPb}\left(\mathrm{I}_{0.5} \mathrm{~B} \mathrm{Br}_{0.5}\right)_{3}$ from DMF. 


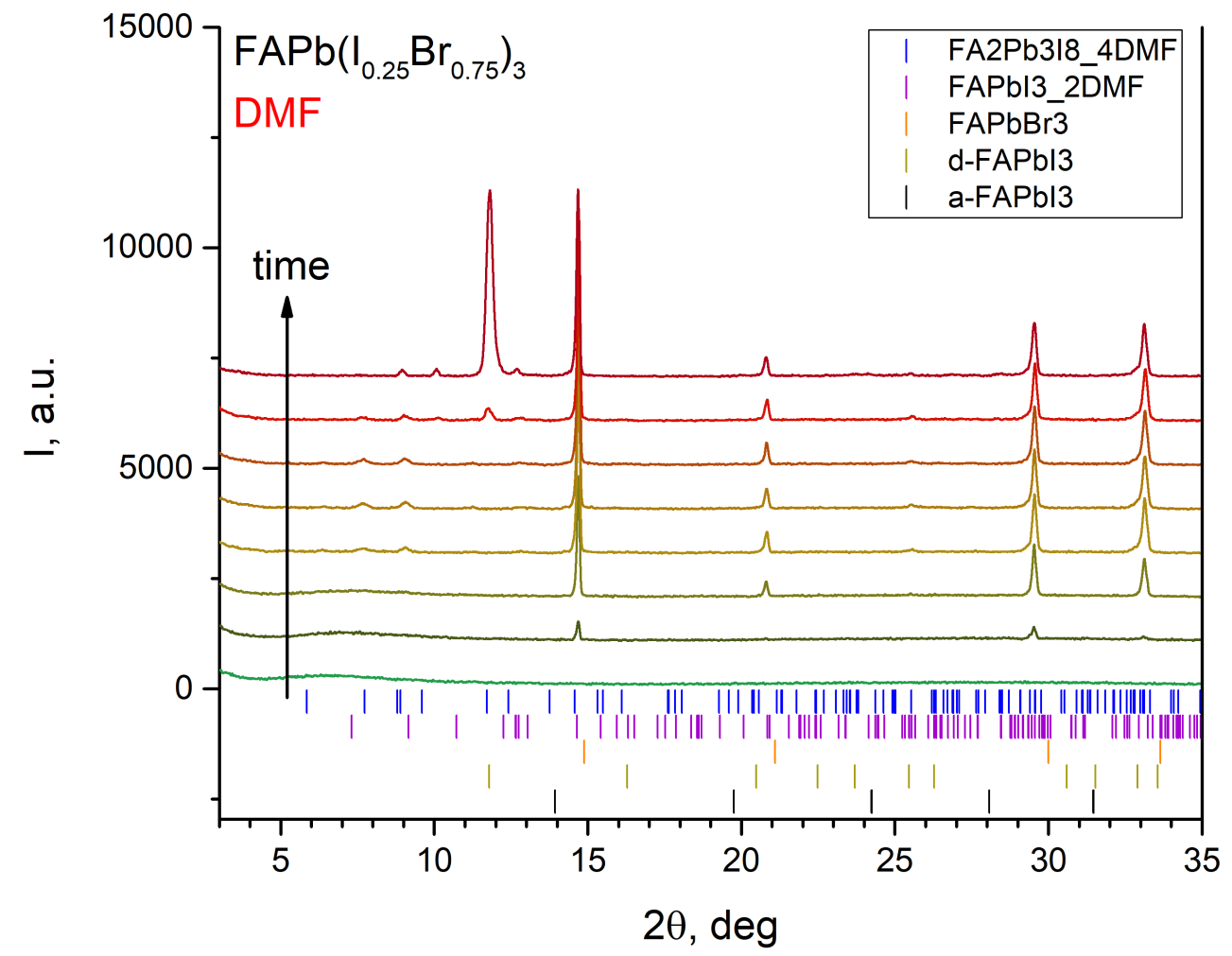

Figure S27. Crystallization of $\mathrm{FAPb}\left(\mathrm{I}_{0.25} \mathrm{Br}_{0.75}\right)_{3}$ from DMF.

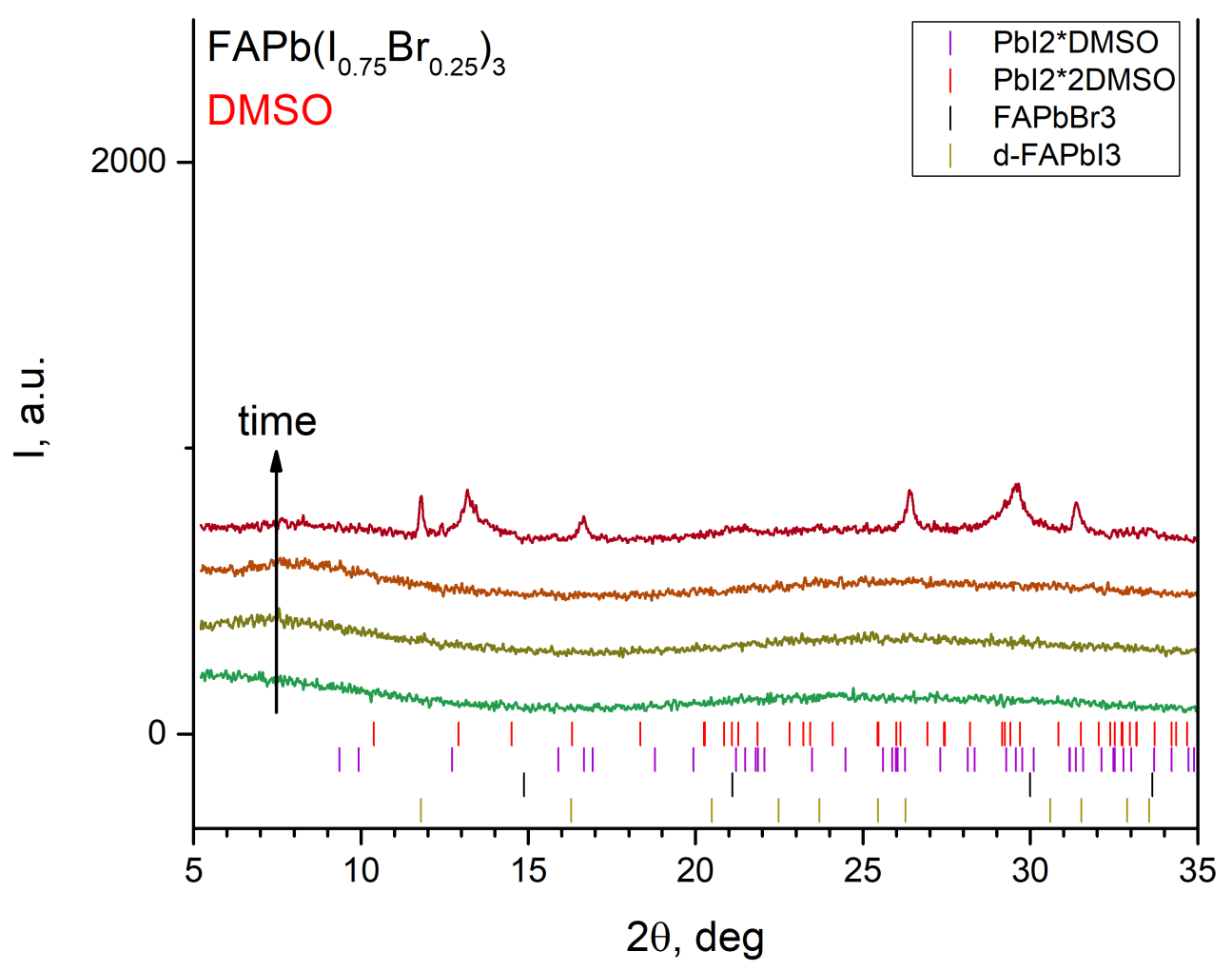

Figure S28. Crystallization of $\mathrm{FAPb}\left(\mathrm{I}_{0.75} \mathrm{Br}_{0.25}\right)_{3}$ from DMSO. 


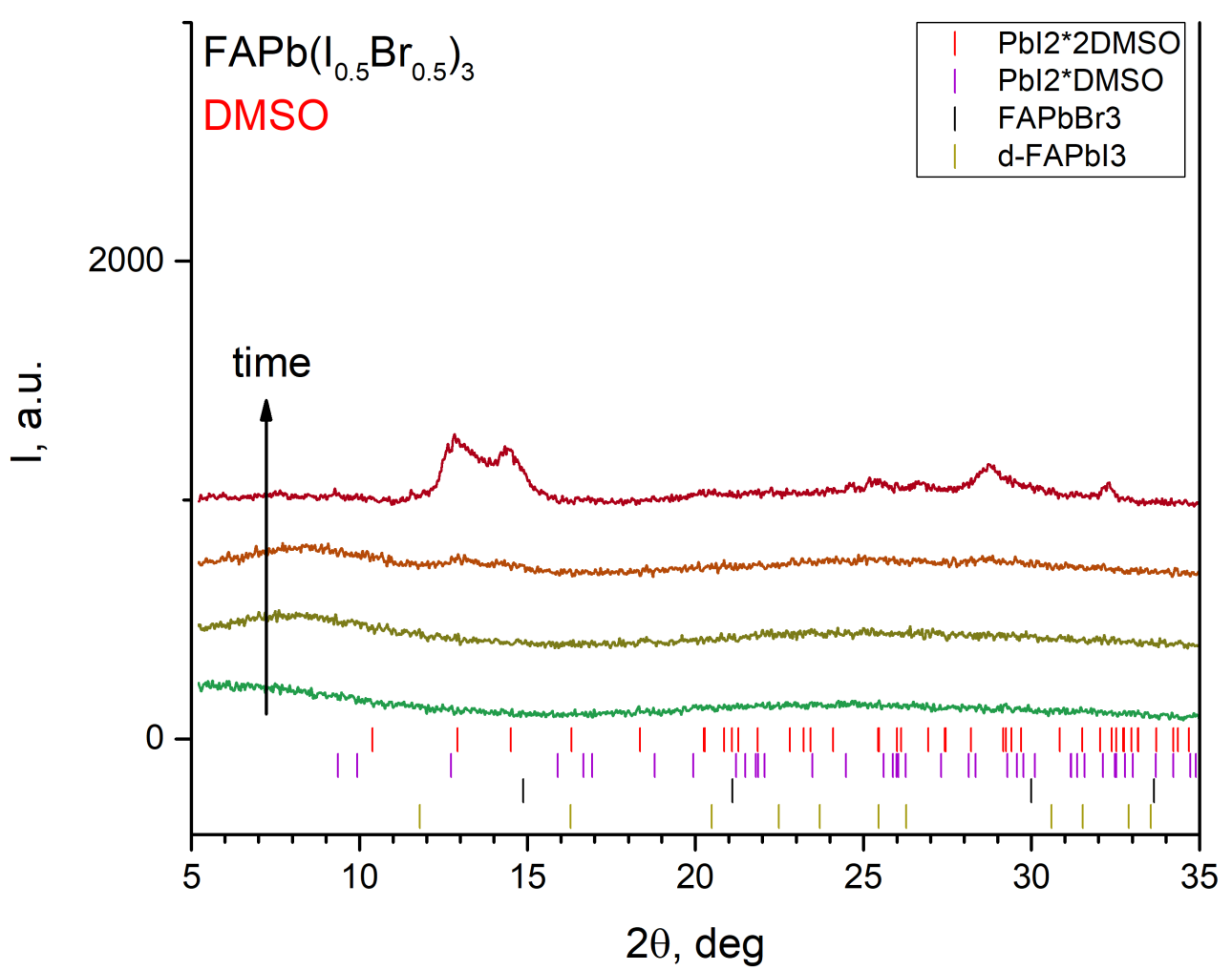

Figure S29. Crystallization of FAPb $\left(\mathrm{I}_{0.5} \mathrm{Br}_{0.5}\right)_{3}$ from DMSO.

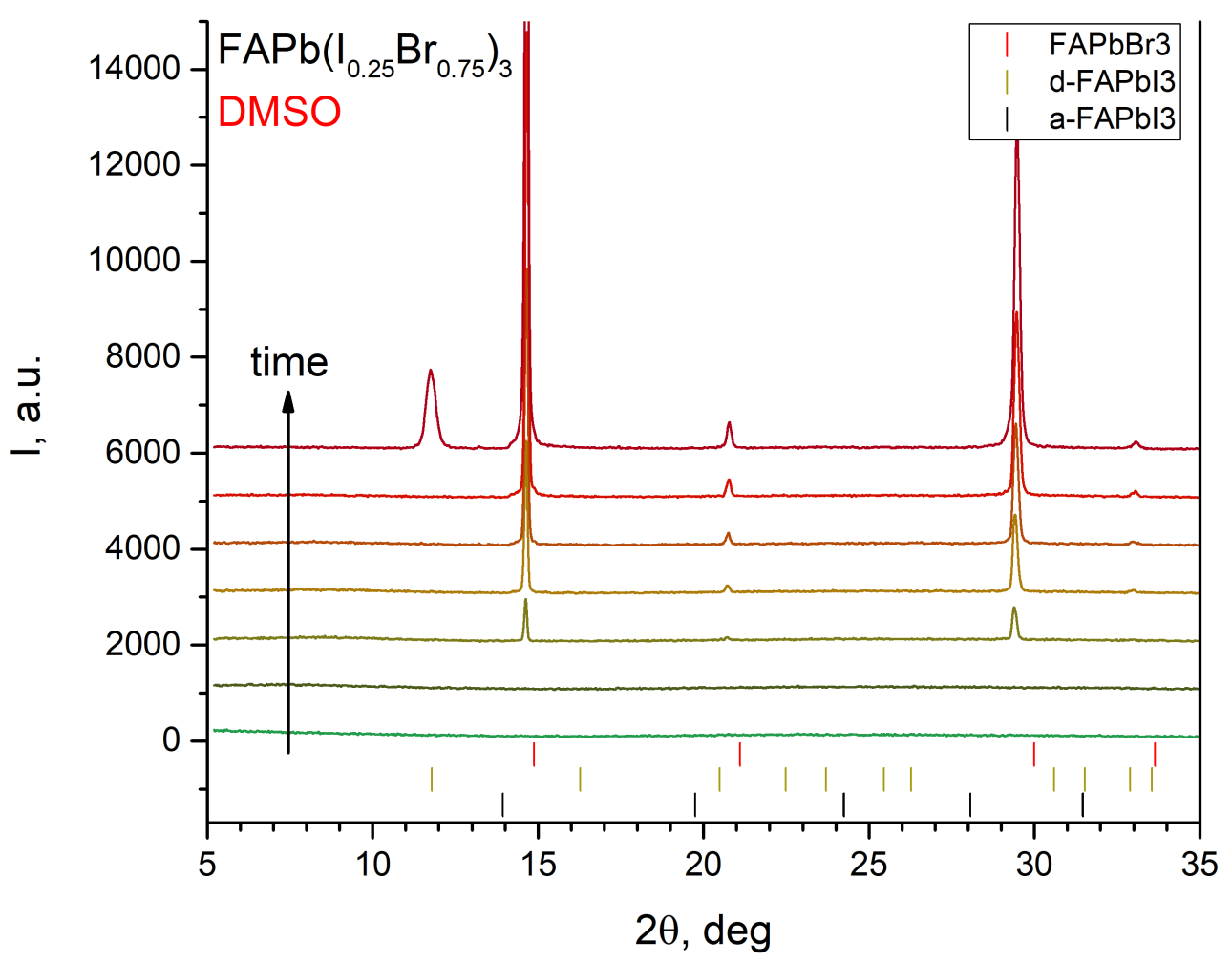

Figure S30. Crystallization of $\mathrm{FAPb}\left(\mathrm{I}_{0.25} \mathrm{Br}_{0.75}\right)_{3}$ from DMSO. 


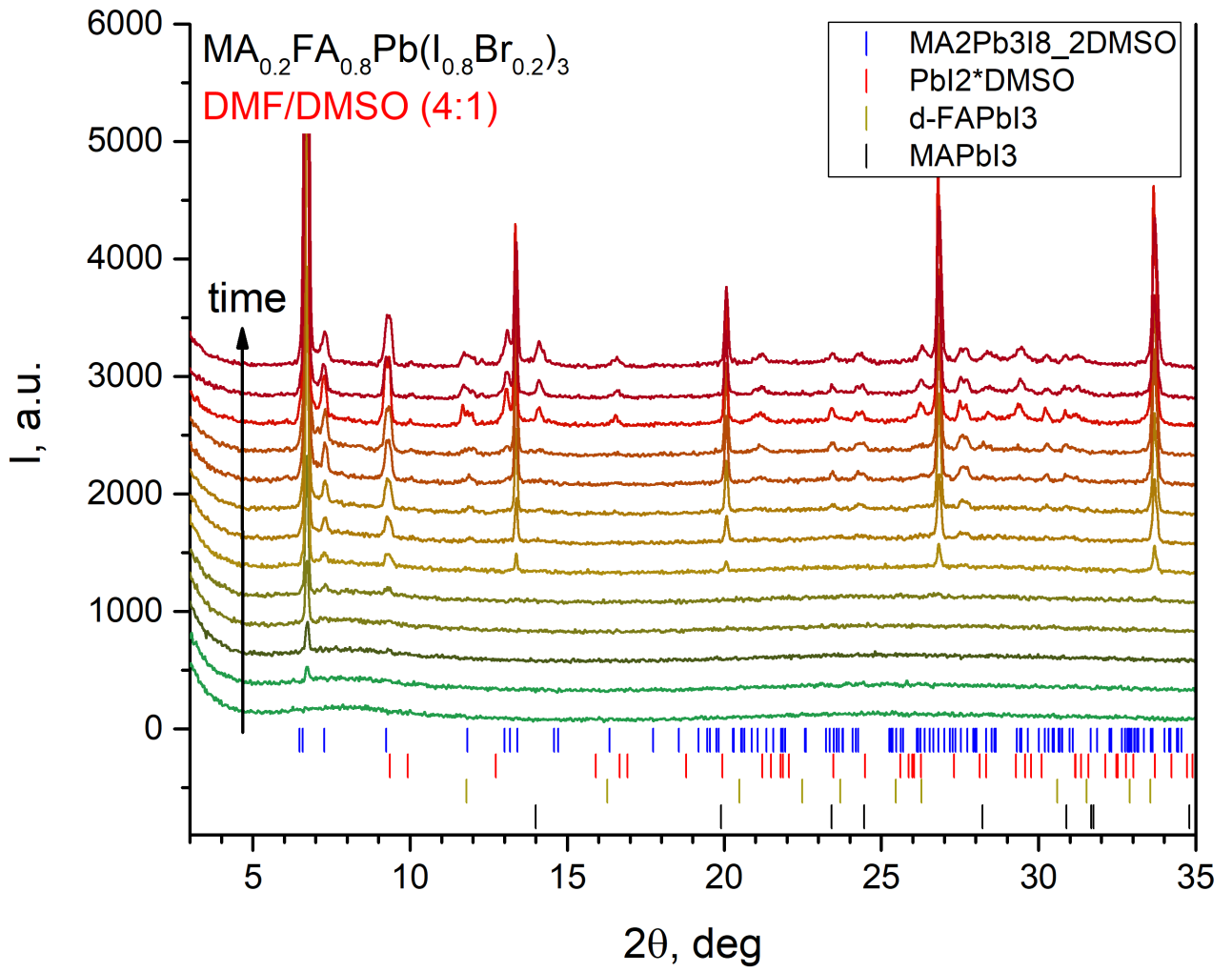

Figure S31. Crystallization of $\mathrm{MA}_{0.2} \mathrm{FA}_{0.8} \mathrm{~Pb}\left(\mathrm{I}_{0.8} \mathrm{Br}_{0.8}\right)_{3}$ from the mixture DMF/DMSO = 4:1. 